\title{
The electron density of Saturn's magnetosphere
}

\author{
M. W. Morooka ${ }^{1}$, R. Modolo ${ }^{1}$, J.-E. Wahlund ${ }^{1}$, M. André ${ }^{1}$, A. I. Eriksson ${ }^{1}$, A. M. Persoon ${ }^{2}$, D. A. Gurnett ${ }^{2}$, \\ W. S. Kurth ${ }^{2}$, A. J. Coates ${ }^{3}$, G. R. Lewis ${ }^{3}$, K. K. Khurana ${ }^{4}$, and M. Dougherty ${ }^{5}$ \\ ${ }^{1}$ Swedish Institute of Space Physics, Uppsala, Sweden \\ ${ }^{2}$ Department of Physics and Astronomy, University of Iowa, Iowa City, IA, USA \\ ${ }^{3}$ Mullard Space Science Laboratory, University College London, London, UK \\ ${ }^{4}$ Institute of Geophysics and Planetary Physics, University of California, Los Angeles, CA, USA \\ ${ }^{5}$ Space and Atmospheric Physics Group, The Blackett Laboratory, Imperial College London, London, UK
}

Received: 12 May 2008 - Revised: 19 June 2009 - Accepted: 20 July 2009 - Published: 29 July 2009

\begin{abstract}
We have investigated statistically the electron density below $5 \mathrm{~cm}^{-3}$ in the magnetosphere of Saturn $\left(7-80 R_{S}\right.$, Saturn radii) using 44 orbits of the floating potential data from the RPWS Langmuir probe (LP) onboard Cassini. The density distribution shows a clear dependence on the distance from the Saturnian rotation axis $\left(\sqrt{X^{2}+Y^{2}}\right)$ as well as on the distance from the equatorial plane $(|Z|)$, indicating a disclike structure. From the characteristics of the density distribution, we have identified three regions: the extension of the plasma disc, the magnetodisc region, and the lobe regions. The plasma disc region is at $L<15$, where $L$ is the radial distance to the equatorial crossing of the dipole magnetic field line, and confined to $|Z|<5 R_{S}$. The magnetodisc is located beyond $L=15$, and its density has a large variability. The variability has quasi-periodic characteristics with a periodicity corresponding to the planetary rotation. For $Z>15 R_{S}$, the magnetospheric density distribution becomes constant in $Z$. However, the density still varies quasi-periodically with the planetary rotation also in this region. In fact, the quasiperiodic variation has been observed all over the magnetosphere beyond $L=15$. The region above $Z=15 R_{S}$ is identified as the lobe region. We also found that the magnetosphere can occasionally move latitudinally under the control of the density in the magnetosphere and the solar wind. From the empirical distributions of the electron densities obtained in this study, we have constructed an electron density model of the Saturnian nightside magnetosphere beyond $7 R_{S}$. The obtained model can well reproduce the observed density distribution, and can thus be useful for magnetospheric modelling studies.
\end{abstract}

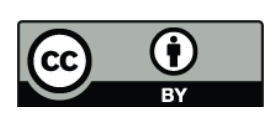

Correspondence to: $\mathrm{M}$. W. Morooka (morooka@irfu.se)
Keywords. Magnetospheric physics (Magnetospheric configuration and dynamics; Planetary magnetospheres; Instruments and techniques)

\section{Introduction}

Observations during the flybys of Pioneer 11 and Voyagers 1 and 2 provided information on plasma conditions in Saturn's magnetosphere. Using observations from the Voyager spacecraft, several plasma density models have been developed to describe the plasma distribution in Saturn's magnetosphere. For the inner magnetosphere, inside $12 R_{S}$, Richardson and Sittler (1990) developed an electron density model in which the density has its peak around the equatorial plane and decreases with increasing L-shell and distance from the equatorial plane. In Saturn's inner magnetosphere, volatile material from the rings and moons are plasma sources and, since the plasma tends to co-rotate with the rapidly rotating planet, the centrifugal force causes the plasma to concentrate near the equatorial plane.

Recent observations have made it possible to study the magnetospheric electron densities in more detail (Moncuquet et al., 2005; Persoon et al., 2005, 2006; Wahlund et al., 2005, Sittler et al., 2008). Persoon et al. (2005) used upper hybrid resonance emissions to measure the equatorial electron density, and found that the electron density varies significantly inside $5 R_{S}$, but consistently drops off exponentially with increasing distance from Saturn beyond $5 R_{S}$. This result implies an ionized material originates from Saturn's moons and is subsequently transported outward via centrifugal forces.

In the outer magnetosphere beyond $12 R_{S}$, Voyager measurements showed that the density is highly variable

Published by Copernicus Publications on behalf of the European Geosciences Union. 
(Richardson et al., 1986). A later density model (Richardson, 1995) showed alternating regions of high- and low- density plasma with increasing L-shell. However, such an alternating density profile is unrealistic and the measurements were more likely affected by some dynamics, as suggested by the authors. Recent observations reveal that Saturn's outer magnetosphere has an asymmetric structure connected to the planetary longitude system. Since Saturn's magnetic dipole is aligned almost parallel to the spin axis of the planet (tilt angle $<1^{\circ}$ ), such periodic behavior of Saturn's magnetosphere has not been expected (e.g., Connerney et al., 1982). On the other hand, many observations clearly demonstrate a spin modulation of the magnetosphere (e.g., Espinosa and Dougherty, 2000; Clarke et al., 2006; Krupp et al., 2005; Paranicas et al., 2005, Carbary et al., 2007, 2008; Krimigis et al., 2007; Gurnett et al., 2007; Arridge et al., 2008). Significant spin asymmetry in the magnetosphere pointed toward additional plasma dynamics, whose origin is in the Kronian magnetosphere itself. Several models have been developed to explain the periodic modulation (Espinosa et al., 2003; Khurana and Schwarzl, 2005; Gurnett et al., 2007; Southwood and Kivelson, 2007), but its origin is still controversial. Gurnett et al. (2007) found a longitudinal asymmetry in the density distribution in the inner magnetosphere, which was unexpected since the Voyager data showed a rather stable density distribution inside $12 R_{S}$, and suggested that this region may be the source of the longitudinal asymmetry in the plasma structure of the outer magnetosphere. On the other hand, Arridge et al. (2008) suggests that the periodic modulation of the particle densities can be due to the periodic vertical motion of the plasmasheet. Such vertical motion can be induced by longitudinally asymmetric field-aligned currents in the inner magnetosphere (Southwood and Kivelson, 2007). Most of the in-situ observations above were made by Cassini in the equatorial plane, while Krupp et al. (2005) found a quasi-periodic modulation of the high-energy $(28-49 \mathrm{keV})$ electrons when the orbit covered the Southern Hemisphere. They compared the electron flux with the magnetic field and concluded that the electron flux modulation was explained by the fact that the spacecraft repetitively entered and exited the plasma sheet and lobe regions due to the modulation of the plasmasheet thickness. To evaluate how well these models of the asymmetry correspond to reality, it is important to investigate the plasma conditions statistically.

In this study, we will use the unique capabilities of the Cassini RPWS Langmuir probe, LP (Gurnett et al., 2004) to measure the plasma density to very low values by means of the spacecraft potential. The LP can measure the electron density, among other plasma parameters, in several different ways. Under outer magnetospheric conditions, where the photoelectron cloud dominates the plasma around the spacecraft, the spacecraft potential $\left(U_{S C}\right)$ and the plasma density are anti-correlated (Escoubet et al., 1997; Pedersen, 1995). This is because the spacecraft must maintain a current balance between the photoelectrons it emits and the plasma elec- trons it collects. Using the floating potential of the probe $\left(U_{\text {float }}\right)$ (Wahlund et al., 2005), which depends directly on the spacecraft potential (Cully et al., 2007), we can obtain the electron number density in Saturn's magnetosphere, as described in Sect. 2 below.

In this paper, we present the electron density inferred from the floating potential data obtained by the LP sensor during 44 orbits through Saturn's magnetosphere. After the successful Saturn orbit insertion (SOI) on 15 July 2004, Cassini has continued to obtain data within and upstream of Saturn's magnetosphere. Up to May 2007, a large part of the magnetospheric equatorial region, mainly the dawn and tail region inside $L=80$, had been covered by Cassini orbits. The orbits also covered the nightside Northern Hemisphere and the dayside Southern Hemisphere. We present the distribution of density with L-value and distance from the equatorial plane $(Z)$.

We use the Kronocentric Magnetic Equatorial (KME) coordinate system, which is a right-handed Cartesian system wherein Saturn as the centre of the coordinate system. The Z-axis points along Saturn's magnetic axis (which is closely aligned to the rotation axis), the $\mathrm{X}$-axis lies in the plane containing the $\mathrm{Z}$-axis and the vector from the centre of the planet to the sun, and $Y$ completes the triad. One may argue that in the outer magnetosphere, the solar wind should be the main determinant for the magnetospheric symmetry, so that the KSM (Kronocentric Solar Magnetospheric) coordinate system, where the Y-axis is the same as in KME but the Xaxis points to the Sun and $\mathrm{Z}$ completes the triad, is more appropriate. However, due to the strong corotation, KME is still a good coordinate system even at the distances we will study, and is also preferable for comparability with studies of the density in Saturn's inner magnetosphere (Persoon et al., 2005, 2006). We also use the L-value, based on a dipole approximation, and SKR longitude system to organize our data. Due to the gaseous nature of the planet, Saturn's rotation has been difficult to measure. A longitude system for Saturn was adopted by the International Astronomical Union (IAU) using the periodic nature of the Saturn kilometric radiation (SKR) (Desch and Kaiser, 1981). Recent observation found a highly stable periodicity of the emission (Giampieri et al., 2005), though a time dependent drift of the periodicity was recognized. Many observations show the modulation in the SKR longitude system. We also used The SKR longitude (SLS3) defined by Kurth et al. (2008) in this study to describe the periodic modulation of the electron density.

The outline of the paper is as follows. In Sect. 2, we establish a relation between the floating potential from the Langmuir probe and the plasma density. In Sect. 3, we use this relation to study the electron density in Saturn's magnetosphere, first for two sample orbits in Sect. 3.1 and then an overall survey of all the 44 orbits used in Sect. 3.2. We consider the detailed statistics for the observed densities near and out of the equatorial plane in Sects. 3.3 and 3.4, respectively. Section 4 is devoted to interpretation, starting in 
Sects. 4.1-4.3 with a discussion of the various regions suggested by the data and proceeding in Sect. 4.4 to model the observed rotating asymmetry. This leads to a final model for the plasma density in Saturn's magnetosphere in Sect. 5. We conclude by a summary in Sect. 6 .

\section{Density estimation by the Langmuir probe}

The Langmuir probe (LP) sensor is designed to provide insitu information of the ambient plasma. When applying a positive or negative bias potential, the spherical Langmuir Probe will attract or repel the electrons and ions from the ambient plasma, and the resulting current is measured. The temperatures and the number density of the electrons and ions in the ambient plasma can be estimated from the probe characteristics, i.e. from how the current depends on the bias potential. Although the LP is well designed to characterize the plasma by direct analysis of the collected plasma particle current in a dense and cold plasma regime (Wahlund et al., 2005), the direct measurement from the probe current becomes difficult in the tenuous plasma. Instead, the photoelectrons generated from the sunlit spacecraft dominate in the space region, and the probe current is balanced by the photoelectrons escaping from the probe and the ambient electrons (the ion contribution is small). In this case, the spacecraft potential relative to ambient plasma can be used to obtain the ambient electron density. Since the LP is mounted rather close $(1.5 \mathrm{~m})$ to the spacecraft body compared to the Debye sheath (can be several hundreds meters in a plasma of $<10^{-1} \mathrm{~cm}^{3}$ and $\sim 100 \mathrm{eV}$ as is typical density and temperature), the floating potential of the LP $\left(U_{\text {float }}\right)$ can be used as a proxy to evaluate the electron number density $\left(N_{e}\right)$. This method can be used when the ambient density falls below $\sim 5 \mathrm{~cm}^{-3}$. To do so, we need to calibrate the measured value of $U_{\text {float }}$ corresponding to the plasma density in some region where both can be determined. We used Electron Spectrometer (CAPS/ELS) (Young et al., 2004; Lewis et al., 2008) data obtained during Cassini Saturn Orbit Insertion (SOI) to calibrate. The ELS is designed to sample the low-energy $(0.58-26000 \mathrm{eV})$ electron population in Saturn's magnetosphere. It is a hemispherical top-hat electrostatic analyzer oriented along the spacecraft $\mathrm{X}$-axis covering $160^{\circ}$ by $5^{\circ}$, and mounted on an actuator, which rotates on the spacecraft's Z-axis through a range of $\pm 104^{\circ}$. As a result, CAPS/ELS can cover approximately $208^{\circ}$ by $160^{\circ}$ of the surrounding spherical space, which is about $56 \%$ of the full $4 \pi$ space. This might lead to an under-estimate or overestimation of the density if the electron population does not have an isotropic Maxwellian distribution. However, this is less likely for the SOI orbit (Lewis et al., 2008). The observed $U_{\text {float }}$, and hence the density, can be overestimated when the LP is located partly in the shadow of the spacecraft. We have corrected the LP density estimate using an empirical function of the spacecraft attitude. Moreover, data

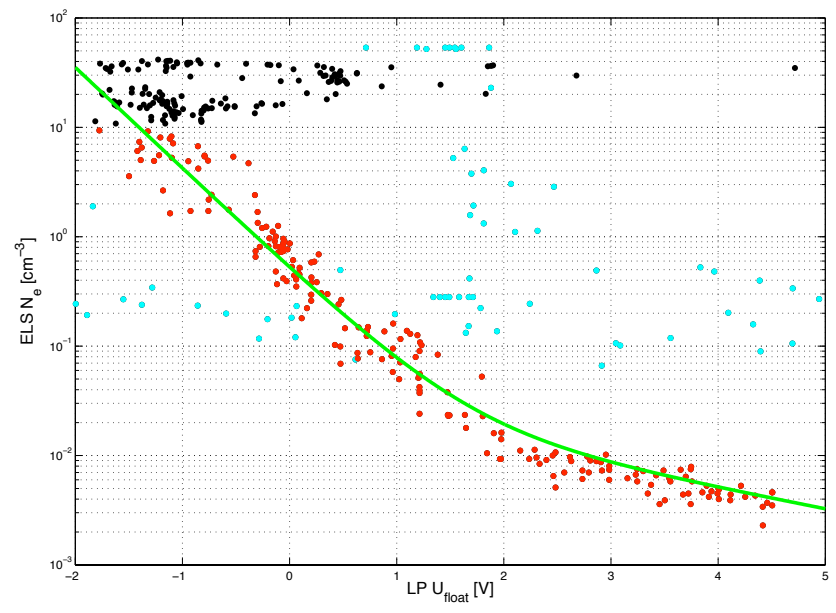

Fig. 1. The relationship between the LP floating potential, $U_{\text {float }}$, and the density measured by ELS, during Saturn orbit injection of Cassini. The data are fitted by two exponential functions using the least squares method. Data with densities above $10 \mathrm{~cm}^{-3}$ are eliminated (black dots). The blue dots indicate data obtained around the ring plane, and are also eliminated from the fitting. Thus, the red circles define the empirical relationship. The empirical fit to the electron density is $\mathrm{Ne}=0.03 \exp \left(-U_{\text {float }} / 2.25\right)+0.50 \exp \left(-U_{\text {float }} / 0.47\right)$ (green line), where $\mathrm{Ne}$ is in $\mathrm{cm}^{-3}$ and $U_{\text {float }}$ in volts.

points where the attitude corrected density differs by more than $50 \%$ from the original density estimate are eliminated. When the LP is in the shadow, the probe current becomes small and our automatic routine to detect $U_{\text {float }}$ often fails. The data are eliminated also in this case.

Figure 1 shows a comparison between the electron densities estimated by CAPS/ELS and the LP floating potential ( $\left.U_{\text {float }}\right)$. The data were sampled from 30th June to 1st July in 2004 when Cassini crossed Saturn's magnetosphere. The data were fitted to two exponential functions using the least square method as

$N_{e}=0.03 \exp \left(-U_{\text {float }} / 2.25\right)+0.50 \exp \left(-U_{\text {float }} / 0.47\right)$

where the density is in $\mathrm{cm}^{-3}$ and $U_{\text {float }}$ is in volts. This expression is a representation of a core and a tail in the photoelectron distribution (Escoubet et al., 1997; Pedersen, 1995; Pedersen et al., 2008). We used densities lower than $10 \mathrm{~cm}^{-3}$ to determine the function. The data obtained around Saturn's ring region (blue dots in Fig. 1) were also eliminated, since very energetic particles from the radiation belts may distort the normal charging behaviour of the spacecraft (Eriksson and Wahlund, 2006). Thus, the red coloured data in Fig. 1 were used for the fit. This proxy relation has an accuracy of about a factor two in absolute density, and only data that give plasma densities smaller than $5 \mathrm{~cm}^{-3}$ using the above calibration have been used in this study. Figure 2 shows a comparison of densities for another orbit in the magnetosphere (from 17 January to 26 January 2006, orbit 20) obtained by ELS (red) and LP (blue and black), the latter using the proxy 


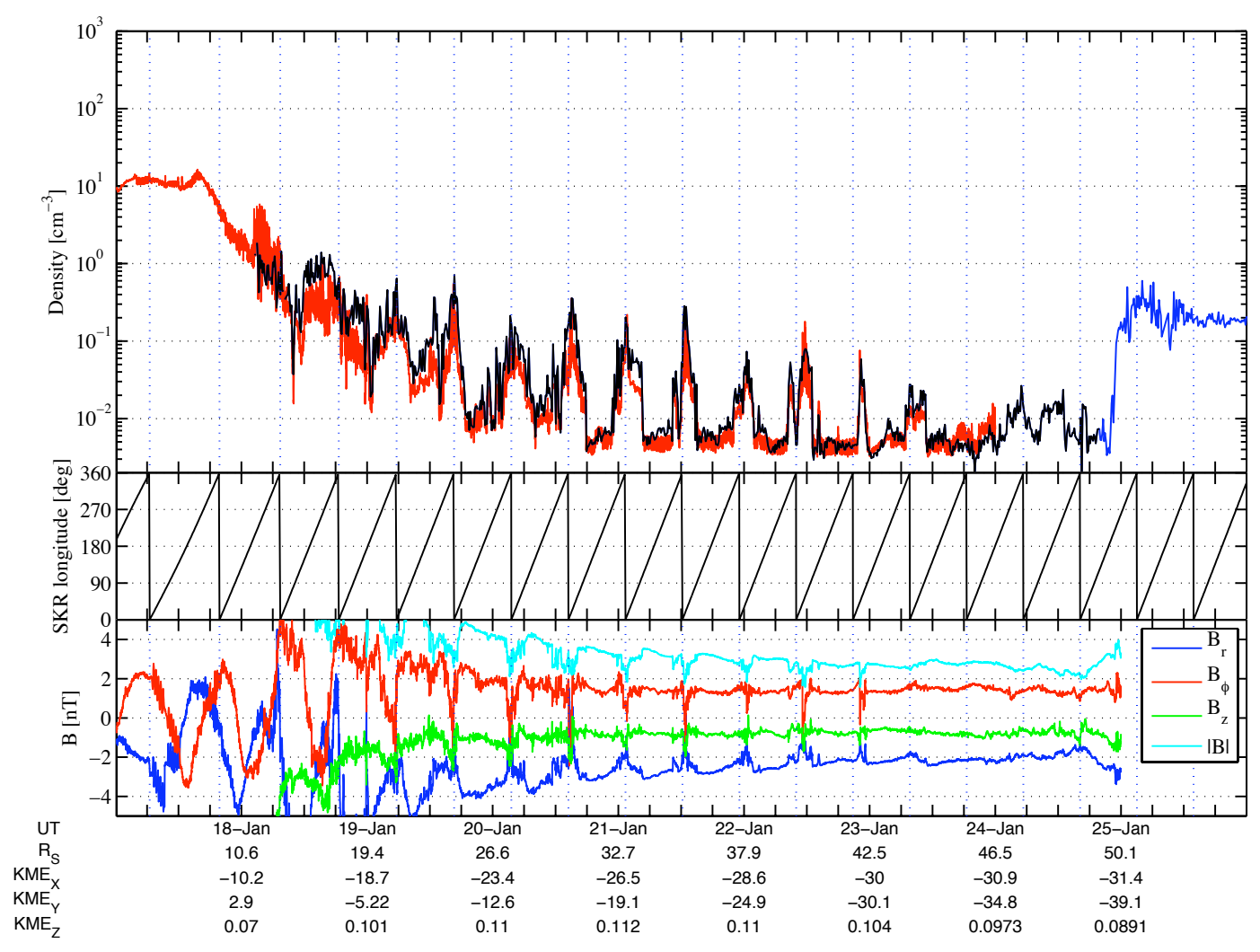

Fig. 2. Top: An example of the densities in the magnetosphere obtained by ELS (red) and the LP proxy method (black and blue) using $U_{\text {float }}$ and Eq. (1). The magnetopuse boundary was identified by a significant increase of the plasma density in the last hours of 24 January, and the data after this time (blue line) have not been used in this study. Thus, only the LP data shown in black are used in this study. Middle: The SKR longitude as defined by Kurth et al. (2007). The time of SKR longitude $=0^{\circ}$ is noted also in the top and bottom panels with blue dashed vertical lines. Bottom: The magnetic field components obtained by MAG. Blue for the radial component, $\left(B_{r}\right)$, red for azimuthal component $(B \phi)$, green for the $\mathrm{Z}$ component $\left(B_{Z}\right)$, and light blue for total magnetic field strength $(|B|)$, respectively. The location of Cassini in the $\mathrm{KME}$ coordinate system is noted below the plot.

function (1). As we wish to investigate the density in Saturn's magnetosphere, time intervals when Cassini was outside the magnetopause have not been considered. This boundary is identified by a significant increase of the plasma density indicative of the magnetosheath. For example, for Cassini orbit 20 (Fig. 2), the magnetopause boundary was detected as a sharp density increase in the last hours of 24 January, and the data after this time (blue line) have not been used in this study. The two data sets agree well after 17 January from 12:00 UT, when Cassini was located at $7 R_{S}$, demonstrating the validity of Eq. (1).

\section{Density in Saturn's magnetosphere}

\subsection{General characteristics}

Figure 2 (upper panel) shows the electron density data obtained by the LP during the time period from 17 January to 26 January 2006. During this time interval, Cassini was situated in the nightside equatorial region travelling from $6 R_{S}$ to $50 R_{S}$ near midnight at a magnetic local time (MLT) of about $3.5 \mathrm{~h}$. The electron density generally decreases with increasing distance from Saturn, and has a periodic variation. The periodic density modulation is clearly seen most of the time. The periodicity was about $10 \mathrm{~h}$ in this case, which is similar to the rotation period of the planet. The SKR longitude (SLS3) defined by Kurth et al. (2008) is shown in the middle panel. The density enhancements appear near the time of SKR longitude $=0^{\circ}$ (the blue dashed vertical lines), suggesting that the density variation is synchronized to the SKR longitude system. It is clear that this density variation is due to a longitudinal asymmetry, with Cassini alternately residing in high and low density regions. The bottom panel shows the magnetic field components in cylindrical coordinates, $B r$ (with $r=\sqrt{X^{2}+Y^{2}}$ ), $B \phi$ (azimuthal direction), $B_{Z}$ (z-direction), and $|B|$ (field magnitude). One notices that decreasing values and orientation changes of the magnetic field are associated with the density enhancements.

Figure 3 shows another example obtained from 25 January to 1 February 2007. During this time, Cassini was located 


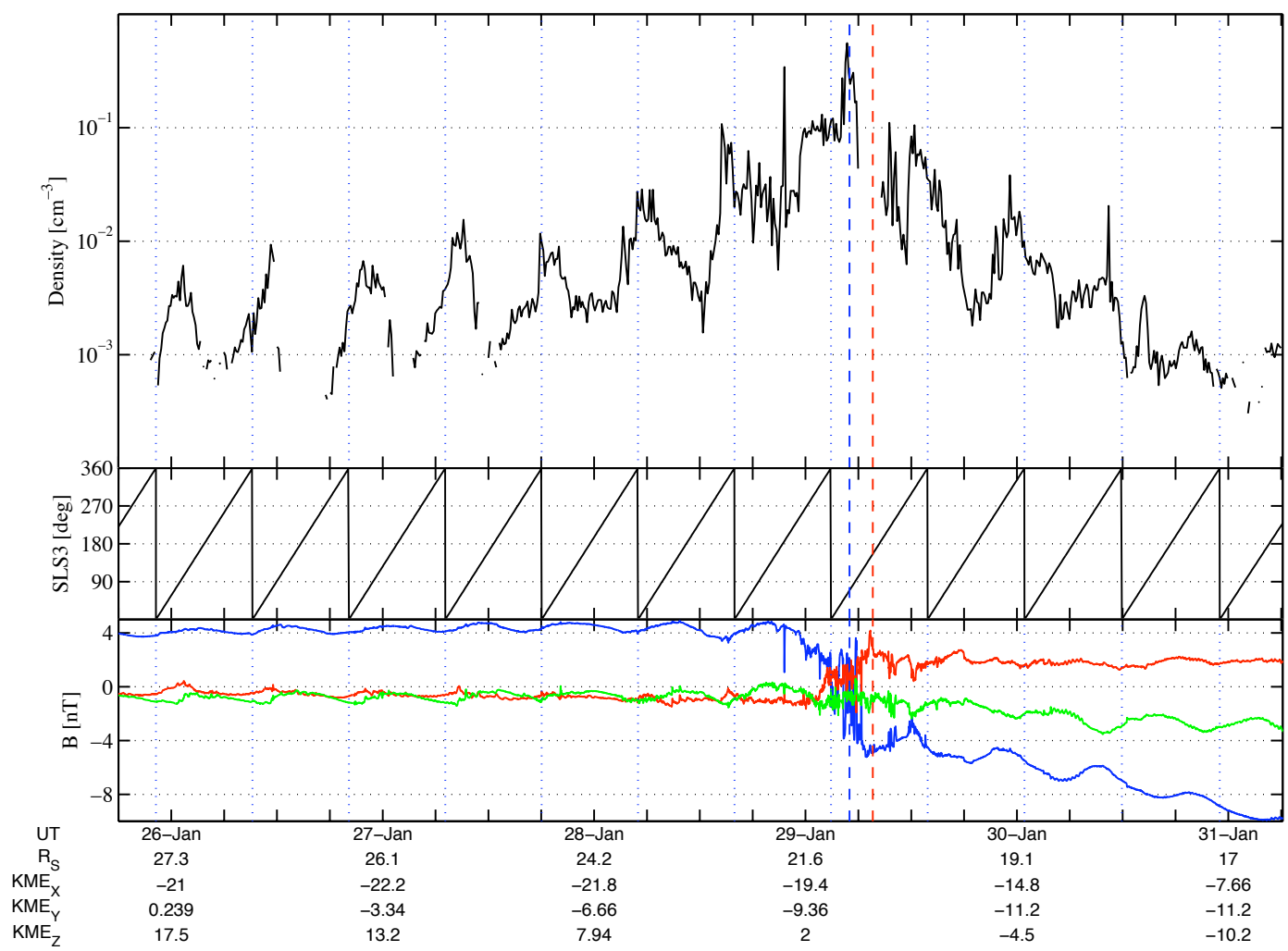

Fig. 3. Top: The electron density data obtained by LP during orbit 38. Middle: The SKR longitude. Bottom: The magnetic field data. The colours are same as for the MAG data in Fig. 2.

in the nightside (MLT=23:00-03:00 h) at a distance of 17$27 R_{S}$ from Saturn, travelling from the Northern Hemisphere to the Southern Hemisphere within the latitude range from $50^{\circ}$ to $-50^{\circ}$. We can see that the electron density generally decreases with increasing distance from the equator $\left(Z_{\mathrm{KME}}\right)$. The density has a periodic variation close to the planetary rotation period. Similar to the case in Fig. 2, the density peaks appear near the SKR longitude $=0^{\circ}$. This periodic signature is observed through the entire plotted interval. The magnetic field perturbation (the bottom panel) is also associated with the periodic modulation of the density. Notice that the maximum of the density (the blue dashed vertical line) appeared just before the time that Cassini was at the equatorial plane (the red vertical line). The $B_{r}$ component of the magnetic field changed from positive to negative at the same time as the density maximum, indicating that the centre of the plasmasheet was slightly northward $\left(0.8 R_{S}\right)$ for this event. Also notice that the density maximum appeared near the time of SKR longitude $=0^{\circ}$.

With the examples shown above, we have obtained two clear qualitative characteristics of the electron density in Saturn's magnetosphere: 1) The electron density generally decreases with increasing distance from the planet and distance from the equatorial plane. 2) The electron density has a periodic variation throughout a large region of the magnetosphere.

\subsection{Statistical studies}

We have used the densities obtained by the above method to construct an electron density map of Saturn's magnetosphere. Figure 4 shows the Cassini trajectories used in this study projected onto the equatorial plane along dipole magnetic field lines, from 27 August 2004 to 2 May 2007. Green lines correspond to the Southern Hemisphere, red lines to the equatorial plane $\left(-0.5 R_{S}<Z<0.5 R_{S}\right)$, and blue lines to the Northern Hemisphere.

Figure 5 shows the densities along the Cassini trajectories in the $\left(\sqrt{X^{2}+Y^{2}}, \mathrm{Z}\right)$ plane. A disc-like feature with high densities $\left(>0.1 \mathrm{~cm}^{-3}\right)$ is clearly visible inside $\sqrt{X^{2}+Y^{2}}=20$ near the equatorial plane for $|Z|<5 R_{S}$. We will identify this inner region as the extension of plasma disc. Outside this inner region, the density is generally lower. Here also the plasma density has a disc-like structure with a region of relatively high-density confined in the magnetic equator region. The plasma densities are very low $\left(<10^{-2} \mathrm{~cm}^{-3}\right)$ at high latitudes both in the Northern $\left(Z>10 R_{S}\right)$ and the Southern $\left(Z<-6 R_{S}\right)$ Hemispheres, while they are relatively high $\left(10^{-2}\right.$ to $\left.10^{-1} \mathrm{~cm}^{-3}\right)$ near the equatorial plane for $-6<Z<10 R_{S}$. This high-density region is not symmetrical with respect to the equatorial plane. This asymmetry is highly interesting, and there are two possible sources. One reason could be that it is an artefact of the local time 


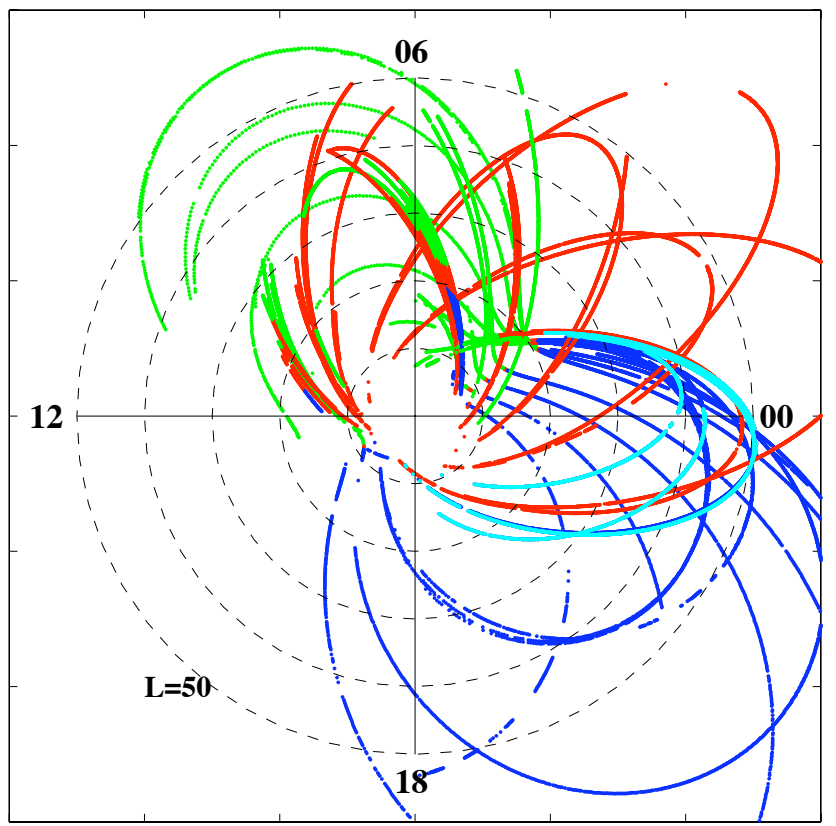

Fig. 4. The orbital distribution of the Cassini data used in this study, projected onto the equatorial plane using a dipole field model, thus illustrating the coverage in $\mathrm{L}$ and local time. The colours indicate the location of Cassini above/below the equatorial plane. The equatorial region $\left(-0.5 R_{S}<Z<0.5 R_{S}\right)$ in red; the Northern Hemisphere is in blue (both light and dark blue); and the Southern Hemisphere is in green, respectively. The light blue indicates orbits 027 , 028, and 029, which were taken between August and October in 2006.

asymmetry of the Cassini orbits (see Fig. 4) combined with a day-night asymmetry of the magnetosphere. In particular, there is a difference between nightside (MLT $>15$ and MLT $<3)$ coverage and dawn dayside $(3<$ MLT $<15)$ coverage. Solar wind pressure on the dayside can induce such dayside/nightside asymmetry. Alternatively, this could be a real asymmetry between the Northern (winter) and Southern (summer) Hemisphere, which could be induced by the solar wind as suggested for the Jovian plasma disc (Khurana and Schwartzl, 2005) or a dipole tilt caused by a relatively simple (though unexplained) field aligned current system in the inner magnetosphere as suggested by Southwood and Kivelson (2007). The actual cause of this asymmetry needs to be reconsidered when a larger Cassini data set becomes available.

Looking at details of the density distribution along each orbit, one can note a quasi-periodic behaviour in the plasma density. Especially in the equatorial region, the density fluctuates by two orders of magnitude. As we have seen in Figs. 2 and 3, these fluctuations are related to the SKR longitude system and are hence corotating with the planet. Such a behaviour was found by Gurnett et al. (2007) for the full plasma in the inner magnetosphere, and by Krimigis et al. (2007) for higher energy particles $(20-50 \mathrm{keV})$ at least out to Titan orbit $\left(20 R_{S}\right)$ : we can now trace it for the plasma density out

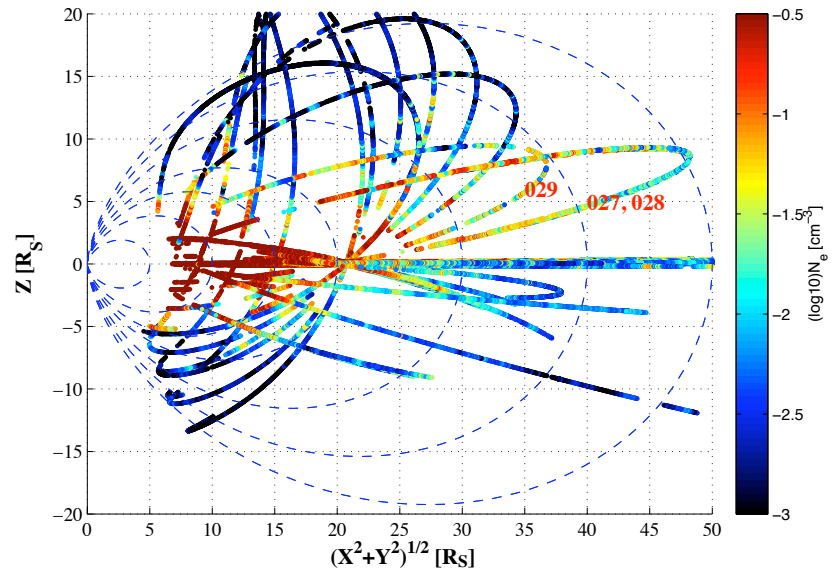

Fig. 5. Electron density along the Cassini trajectory in $\sqrt{X^{2}+Y^{2}}$ and $Z$. The density is colour-coded according to the colour bar displayed to the right in the lower panel. The numbers indicate the three specific orbits shown in light blue in Fig. 3. Blue dashed lines show the dipole field lines for $L=5,10,15,20,30,40$, and 50 .

to more than $30 R_{S}$. The periodic density oscillations can be seen over large regions of the magnetosphere for all of the 44 orbits in this study.

\subsection{Densities in the equatorial region}

Figure 6 shows the density profile with distance from the rotation axis, $\sqrt{X^{2}+Y^{2}}$, in the equatorial region $(|Z|<$ $\left.0.5 R_{S}\right)$. The top and the middle panels are scatter plots of all observed data on the dayside $(8<\mathrm{MLT}<16)$ and the nightside $(20<M L T<4)$, respectively. Black and red dots are the densities obtained by the LP and green dots are the density determined by the RPWS using the upper hybrid wave frequency $\left(f_{U H}\right)$ (Persoon et al., 2005). Comparing the density on the dayside to the one on the nightside, the density on the dayside overall seems to be smaller than that on the nightside. One reason can be that we avoided data when the spacecraft repetitively crossed the magnetopause. A typical location of the magnetopause can be 18 to $30 R_{S}$ (Arridge et al., 2006) where we have most of the data on the dayside. We may then have missed some periods of higher density in the magnetosphere.

It is clear that the density decreases as $\sqrt{X^{2}+Y^{2}}$ increases for all values beyond $5 R_{S}$. Between $7 R_{S}$ and $10 R_{S}$ the LP and the $f_{U H}$ densities (green dots) agree. This agreement between the two independently derived densities further validates the method of using the $U_{\text {float }}$ proxy method for estimating the plasma density. Note that the proxy method is limited to densities below $5 \mathrm{~cm}^{-3}$. The density distribution trend continues from the inner magnetosphere $\left(5 R_{S}\right)$ to the outer magnetosphere beyond $20 R_{S}$. Near Saturn, inside $10 R_{S}$, from the observation by $f_{U H}$, the density variation is rather small. The variation range is less than one order of magnitude. On the other hand, beyond $10 R_{S}$ using the LP 


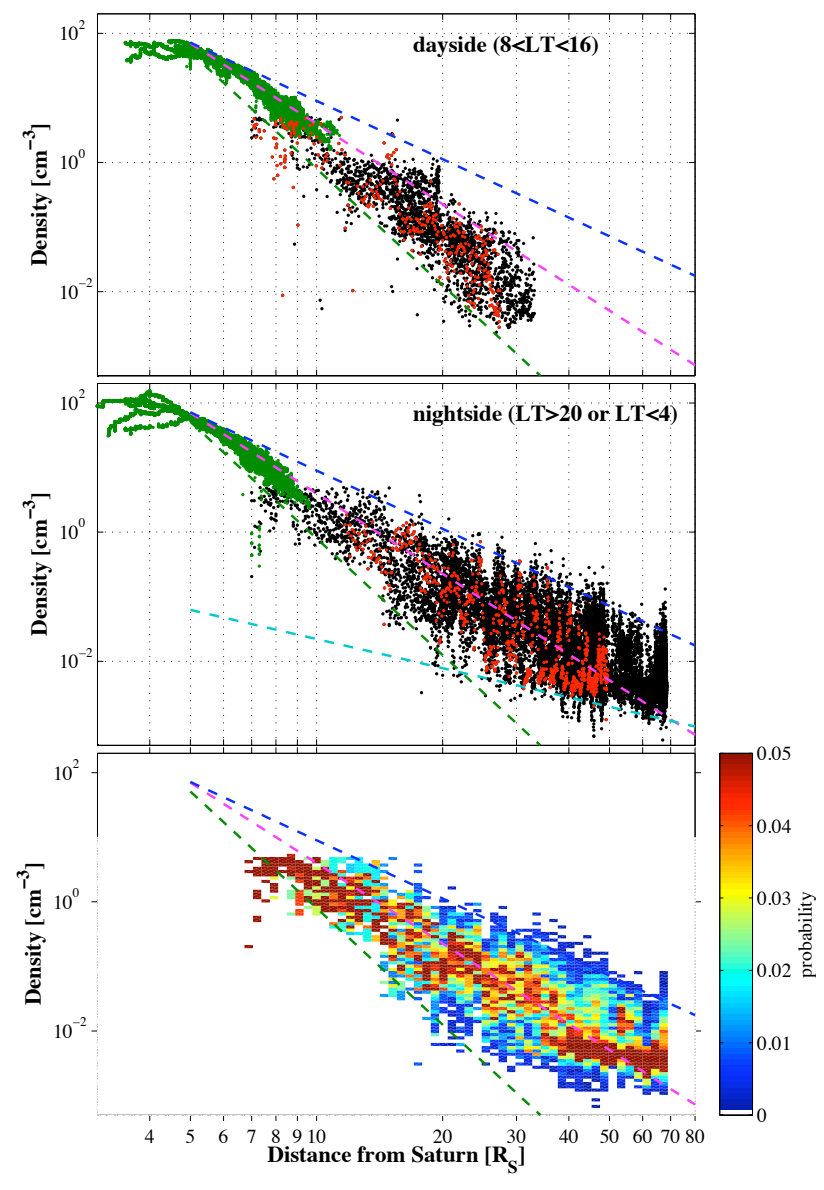

Fig. 6. Density distributions in the equatorial plane $\left(-0.5 R_{S}<\right.$ $Z<0.5 R_{S}$ ). Scatter plots of all the data are shown at the top for the dayside and in the middle for the nightside. The black dots are data by the LP proxy method, and the green dots are derived from $f_{U H}$. The red dots are data by the LP proxy method for the particular orbit obtained from orbit 19 (upper panel, dayside), and from orbit 20 (middle panel, nightside). The bottom panel shows the probability distribution of the density in each L-shell bin in the nightside. The dashed lines in each panel represent the upper limit of the density in the outer magnetosphere (blue line, varies as $810^{3} \mathrm{~L}^{-3}$ ) and the corresponding lower limit (green line, varies as $610^{6} \mathrm{~L}^{-7}$ ). In the distant magnetosphere beyond $\mathrm{L}>30$, the lower limit line becomes $710^{-1} \mathrm{~L}^{-1.5}$ (light blue). The magenta line is the electron density extracted from the model by Persoon et al. (2005), which varies as $5.510^{4} \mathrm{~L}^{-4.14}$.

observations, the variability increases with $\sqrt{X^{2}+Y^{2}}$. Almost all the data can be confined within the upper (blue) and the lower (green) limiting lines, which have dependencies of $L^{-3}$ and $L^{-7}$, respectively $\left(\sqrt{X^{2}+Y^{2}}\right.$ is equivalent to $\mathrm{L}$-value in the equatorial plane). In the distant tail $\sqrt{X^{2}+Y^{2}}>30 R_{S}$, the lower limit line is replaced by the function of $L^{-1.5}$ (dashed light blue line in the middle panel).

The bottom panel in Fig. 6 shows the probability of the density on the nightside in each $\sqrt{X^{2}+Y^{2}}$ range, defined by dividing the number of data points for each logarithmically spaced density interval by the total number of observations in each $\sqrt{X^{2}+Y^{2}}$ bin. The magenta line represents the density model extracted from Persoon et al. (2005) using $f_{U H}$ measurements in which the density varies approximately as $L^{-4}$. High probability appears at the density around and lower than the line of the density model by Persoon et al. (2005). As an example of a particular orbit, the red dots in the upper and the middle panels are the densities obtained from orbit 20 (the same data as the top panel of Fig. 2) on the nightside, and from orbit 19 on the dayside (obtained from 22 to 25 December 2005). On the dayside as well as on the nightside, the density has a trend decreasing with increasing $\sqrt{X^{2}+Y^{2}}$ with a large variation. We showed in Fig. 2 that this variation has a periodic variation connected to the SKR longitude system. The density variation for orbit 19 (not shown here) was also related to the SKR longitude system. Considering the electron distribution in L-shell from the statistics and the longitudinal characteristics obtained from orbit 19 and 20, we expect the densities to be confined between the upper and lower limits defined as $L^{-3}$ and $L^{-7}$ and to vary with longitude. As a result, the mean values of the density follow $L^{-4}$ as was expected by Persoon et al. (2006). Some data obtained inside $15 R_{S}$ show densities below the lower limit. These low-density values may be related to the magnetic flux tube interchange events that are observed in this region of the magnetosphere (Andre et al., 2007; Burch et al., 2007). Note that the upper and the lower lines also well represent the upper and lower density limits of the $f_{U H}$ inside $10 R_{S}$. Although the density is rather stable near Saturn, the density variations gradually increase beyond $7 R_{S}$.

\subsection{Z dependence}

Figure 7 shows the $Z$ dependence of the electron density at $L<16$ on the dayside (upper panel) and the nightside (lower panel). The different colours show sorting with L-shell: blue is for $7<L<12$, green is for $12<L<14$, and red is for $14<L<16$. Under the centrifugal force, the plasma density distribution can be described as $n_{e}(Z)=n_{0} \exp \left[-(Z / H)^{2}\right]$ (Hill and Michel, 1976), where $\mathrm{H}$ is the plasma scale height, and $n_{0}$ is the electron density. Dashed lines are the functions fitted to $n_{0} \exp \left(-(Z / H)^{2}\right)$ using the least square method. The best-fit functions are displayed on the right bottom of each panel. On the dayside $n_{0}$ varies for the $L$ ranges described above as $1.72,0.47$, and 0.32 , and $H$ varies as 2.5 , 3.3 , and 3.6. On the nightside, $n_{0}$ varies as $2.36,0.54$, and 0.26 , and $H$ varies as $2.3,2.5$, and 2.8. Comparing the fitted values, we find, both on the dayside and on the nightside, that $n_{0}$ decreases with increasing L-values, and the plasma scale height $H$ increases with increasing $L$. Although there are not so many data points on the dayside, it seems that the height scale $\mathrm{H}$ is larger than on the nightside. This characteristic is consistent with the high-energy particle observation (Krimigis et al., 2007), suggesting that the dayside plasmasheet extends wider in $Z$ due to the solar wind pressure. 

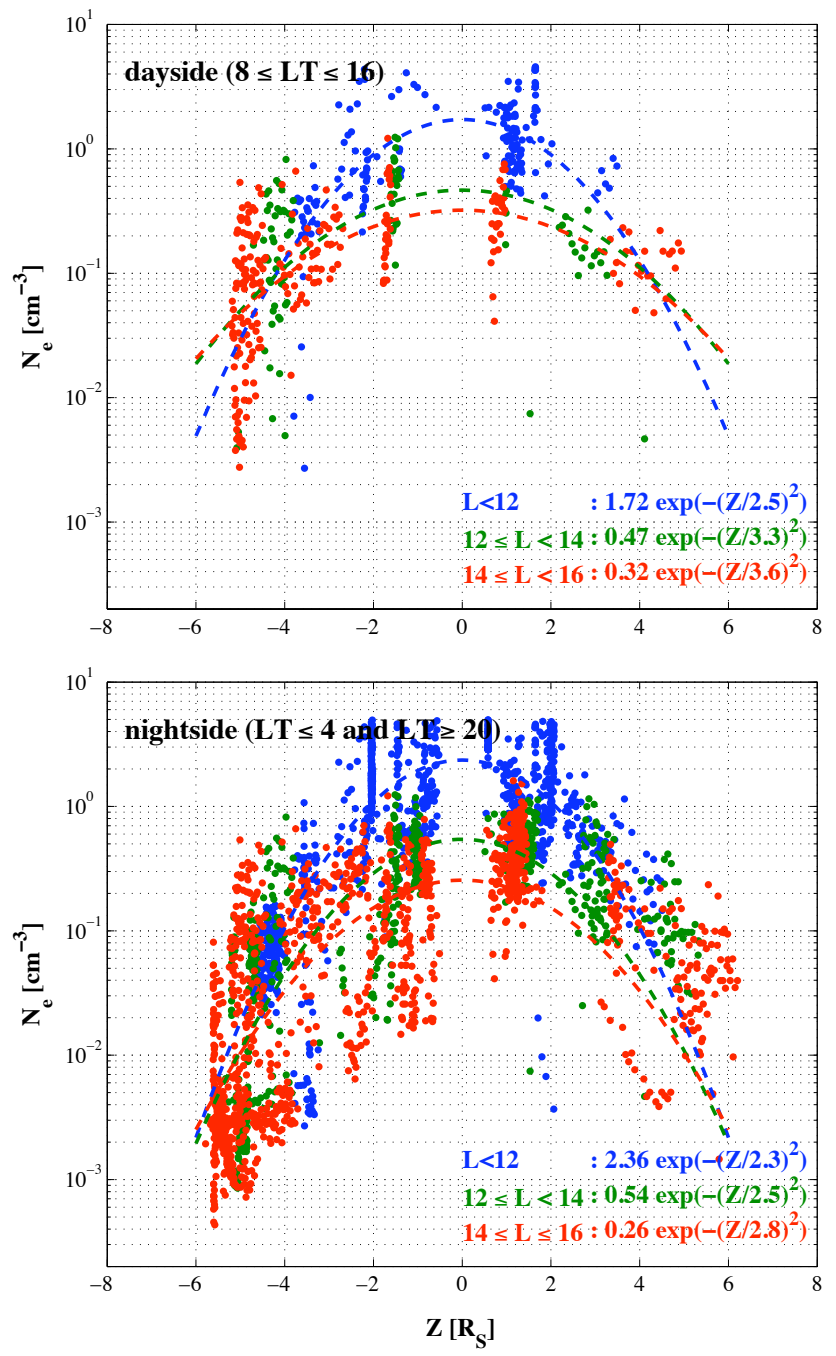

Fig. 7. Density distribution with $Z$ for dipole L-value between 7 and 16 on the dayside (upper panel) and the nightside (bottom panel). Different colours indicate various $L$ values ranges. Blue is for $7<L<12$, green is for $12<L<14$, and red is for $14<L<16$, respectively. Dashed lines indicate functions $n_{0} \exp \left(-(Z / H)^{2}\right)$ fitted to the data using the least square method, as displayed at the bottom right in each panel.

Figure 8a shows the density profile with $Z$ for $L>15$ on the dayside (left panel), dawn side (blue dots on the right panel), and the dusk side (red dots on the right panel), respectively. It is impossible to identify the density maximum point for each local time due to the orbital coverage of Cassini, however, the density overall is larger near the equatorial plane. It is also difficult to define a general trend, since the amount of data is restricted and the density has a large variability. In the dawn region the density above $Z=-5 R_{S}$ has a large variability and the gradient is similar to the one in the nightside near Saturn, which will be shown later. The density variability below $Z=-5 R_{S}$ is small in the dawn region. It seems that the density variation is especially large in the dusk region. On the dayside it seems that the density values and variability are large over a large region. This could be due to the solar wind compression (Krimigis et al., 2007). We have avoided the cases where the spacecraft repetitively crossed the magnetopause, and since it is known that the magnetopause location is periodically changing due to internal dynamics of Saturn's magnetosphere (Clarke et al., 2006), perhaps some data points in the magnetosphere might be missed in our study. The electron density on the dayside must be investigated in more detail with consideration of the repetitive dynamics of the magnetopause.

Figure $8 \mathrm{~b}$ shows the density profile with $Z$ for $L>15$ on the nightside sorted by the L-values (panels a and $\mathrm{c}$ for $L<25$ and panels (b) and (d) for $L>=25$ ). The magenta dots over-plotted in each panel are data taken from orbit 38 (Fig. 3). Overall, the plots show a density maximum near the equatorial plane $(Z=0)$, and a large variability. Comparing all the data to the specific data from orbit 38 , it is clear that the density variability is not due to the local time but rather due to a time dependence, which relates to SKR longitudinal system. At $L<25$, the density decreases with $|Z|$ and has a peak close to the equatorial plane. There are no observations in the Southern Hemisphere for $L>25$, but we see a strong trend that the density decreases with increasing $Z$ in the Northern Hemisphere out to about $Z=15 R_{S}$. From this, we expect that the density maximum should be located near the equatorial plane (at least not in the Northern Hemisphere). Panels (c) and (d) show the probability of the density on the nightside, for bins spaced linearly in $Z$ and logarithmically in density. The number of data points obtained for a certain density value and $Z$ is normalized by the total number of data points for each $Z$. The density value in the high probability density region shows a clear exponential decrease with increasing $Z$. Comparing the high probability region to the data obtained during orbit 38 (magenta in panels a and b), we find a linearly decreasing density with a periodic variability superimposed. The red and the green dots show the highest probability for each $Z$ in the Northern Hemisphere and the Southern Hemispheres, respectively. Using these peaks, the density distribution at $L<25$ was fitted as $0.530 \exp \left(-(Z-1)^{0.78}\right)$ for the Southern Hemisphere and $0.194 \exp \left(-(Z-1)^{0.75}\right)$ for the Northern Hemisphere, respectively. The density distribution beyond $L=25$ was fitted as $0.242 \exp \left(-(Z-1)^{0.61}\right)$ using the data from $Z<=15$. The density peak location was slightly above the equatorial plane at $Z \approx 1$ for $L<25$. Therefore the densities are fitted using a northward shift. The gradient of the fitted curves do not have significant differences between the Northern and the Southern Hemispheres inside $L=25$.

For most $Z$ values in the nightside beyond $L=25$, the maximum probability is found along the fitted lines. However, around $Z=7-10 R_{S}$, the maximum probability instead appears at much higher density. We will discuss the significance of this observation below. 

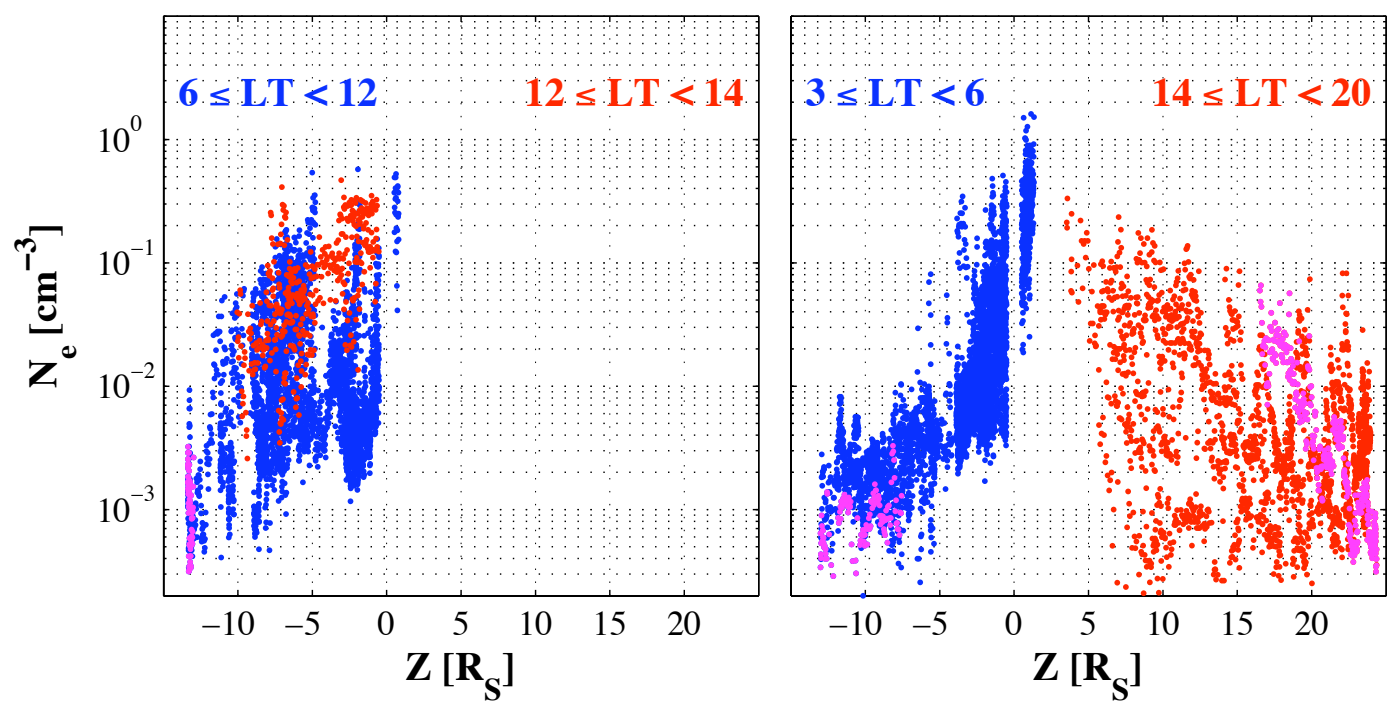

Fig. 8a. Density distribution with $Z$ beyond $L=15$ on the dayside. Left: the dayside $(6<=\mathrm{MLT}<14)$ data. Right: the data from the dawn and the dusk side.
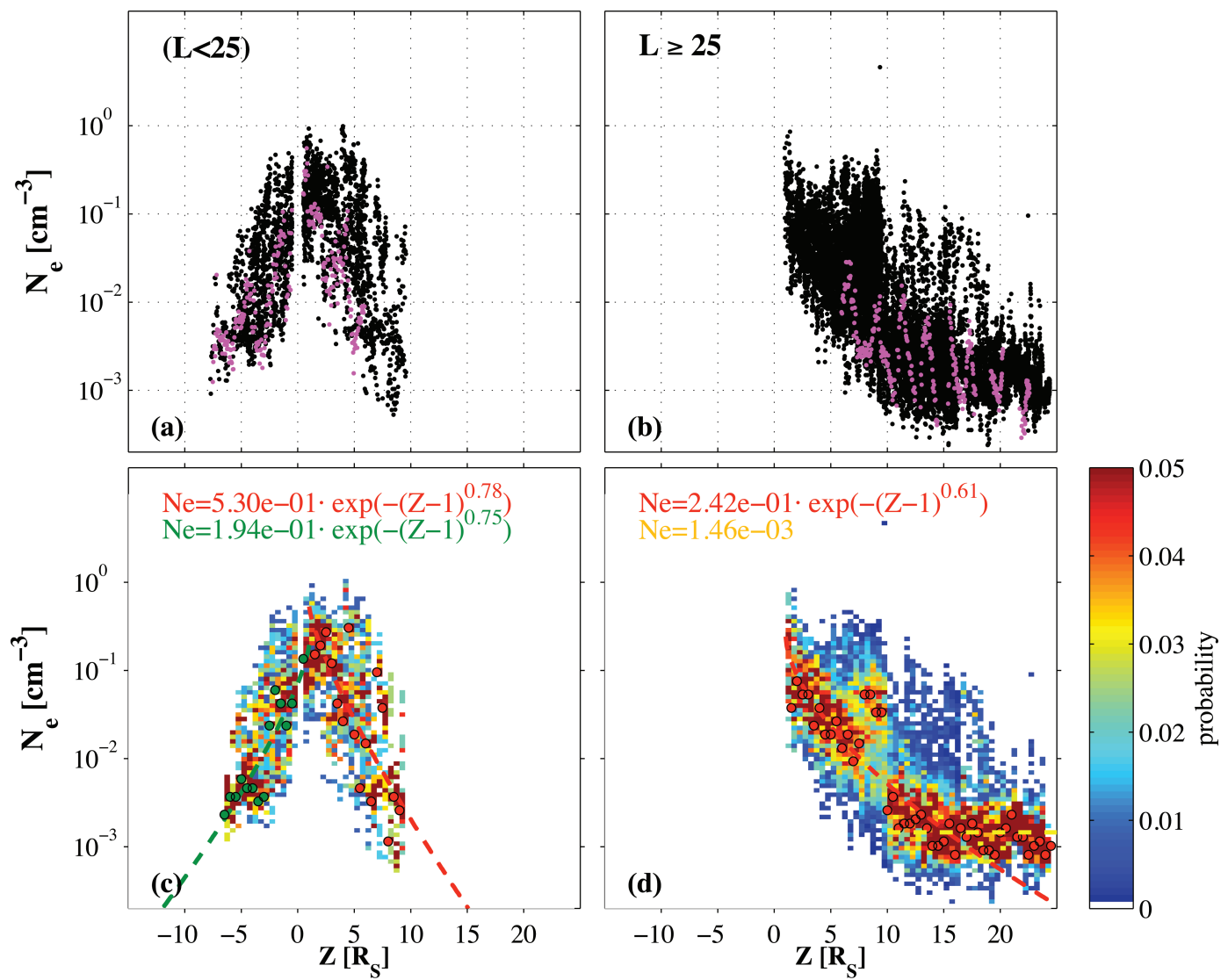

Fig. 8b. (a) Density distribution with $Z$ at $15 \leq L<25$ on the nightside (b) Density distribution with $Z$ beyond $L=25$. Magenta dots are data from orbit 38 (Fig. 3). (c) The probability distribution of the density at $15 \leq L<25$. (d) The probability distribution of the density beyond $L=25$. The red and the green dots in panels (c) and (d) indicate the highest probability for each $Z$. These peaks are used to derive the density distribution in $Z$. For the Northern Hemisphere, only the data at $Z<15 R$ are used to fit the red dashed curve. The data above $Z>15 R_{S}$ are used to derive the average value at $Z>15 R_{S}$ (yellow dashed line). 
Above $Z=15 R_{S}$ the density becomes constant in $Z$. While it should be noted that the relation between floating potential and plasma density shown in Fig. 1 does not extend to densities lower than $310^{-3} \mathrm{~cm}^{-3}$, the physical basis for this relation (spacecraft current balance, see Pedersen, 1995) ensures that it must apply at least approximately also to the lower density values we find here. Therefore, we interpret this lack of $Z$ dependence in the density as a real effect. The average value at $Z>15 R_{S}$ is $1.4610^{-3}$ (yellow dashed line in Fig. $8 \mathrm{~b}$ panel d). Here, the periodic density variations still exist. The magenta dots in panel (b) of Fig. $8 \mathrm{~b}$ are the data from a single event, orbit 38. Comparing the data variability in $Z$ (Fig. 8b) and time (Fig. 3), one understands that the large density variability in the large $Z$ region is also due to the planetary rotational variation. Indeed the density variability is high all over the magnetosphere beyond $L=15$.

\section{Data interpretation and modelling}

Using the proxy relationship between the floating potential measured by the Cassini RPWS Langmuir probe and the plasma density by the ELS instrument (Fig. 1), we have obtained a good estimate of the electron density in a tenous plasma regime $\left(N_{e}<5 \mathrm{~cm}^{-3}\right)$. We have shown that the density thus estimated from the $U_{\text {float }}$ is consistent with the density independently measured by the electron detector (ELS) at another time interval, not used for the calibration (Fig. 2), and with the densities obtained from the RPWS upper hybrid wave emission observations $\left(f_{U H}\right.$, Fig. 6). This LP proxy method has the advantages of not being restricted by the pitch-angle coverage of the instrument, the energy range of the particles or by the intensity of the plasma emissions. Furthermore, the LP data can be sampled with high time resolution of up to $\sim 1 / 16$ sample/second. On the other hand, the proxy method potentially has some sensitivity to spacecraft charging caused by energetic particle events. We have attempted to avoid such events by excluding the ring current region, apparently successfully, as there are little variations suggestive of charging events to be seen in the data.

Using the LP data onboard Cassini, the electron density in Saturn's magnetosphere beyond $7 R_{S}$ has been statistically investigated. We have investigated the electron distribution with increasing L-shell and with distance above and below the equatorial plane. Although we obtained some data on the dayside, the dayside coverage is restricted by the Cassini trajectory and our exclusion of repetitive magnetopause crossing cases. On the nightside, we note the following characteristics:

- In a large region of Saturn's magnetosphere, the electron density has a disc-like distribution, in which $N_{e}$ decreases with increasing $L$ and $|Z|$.
- Inside $15 R_{S}$ within $|Z|<\sim 5 R_{S}$, the electron densities are large $\left(>0.1 \mathrm{~cm}^{-3}\right)$ and depend on $n_{0}$ $\exp \left(-(Z / H)^{2}\right)$.

- Beyond $L=15, N_{e}$ is highly variable and can be divided into two longitudinal sectors: a low-density and a high-density sector. The density in the high-density sector is usually one to two orders of magnitude larger than the density in the low-density sector. The highdensity sector appears periodic with a period similar to the SKR modulation period, demonstrating the character of a longitudinal asymmetry.

- In the equatorial region $\left(|Z|<0.5 R_{S}\right)$ beyond $L=15$, the densities are confined within two limit lines, which decay with distance approximately as $L^{-3}$ and $L^{-7}$. The density varies within the two lines periodically with the planetary rotation. The periodic density variation related to the planetary rotation stays within the two lines. The averaged distribution of the density appears to follow the model density extrapolated from Persoon et al. (2006). The lower limit becomes proportional to $L^{-1.5}$ in the distant magnetosphere beyond $L>30$.

- The densities have a $Z$ dependence of approximately $\exp \left(-Z^{\sim 1 / 2}\right)$, but the gradient might decrease with the $\mathrm{L}$-value. The density peak appears close to the equatorial plane.

- The density distribution becomes constant with $Z$ for $Z>15 R_{S}$, however, the periodic variations still exist due to the planetary rotation and display a clear longitudinal plasma structure.

From the results obtained above, the density in Saturn's magnetosphere can be divided into two longitudinal sectors, corotating with the planet:

- The low-density longitudinal sector where the density scales roughly as $L^{-6}$ and $L^{-1.5}$ at $L>30$.

- The enhanced-density longitudinal sector where the density maxima scales roughly as $L^{-3}$.

For both low and enhanced density sector, the $Z$ distribution depends on $n_{0} \exp \left(-(Z / H)^{2}\right)$ at $L<15$, and $\exp \left(-Z^{0.5}\right)$ at $L>15$. The density becomes extremely low and constant in $Z$ at $Z>15 R_{S}$.

In the following Sects. 4.1-4.3, we summarize our findings for each region of the Saturnian magnetosphere that we have identified in the data. This synthesis forms the basis for a model of the plasma density dependence on $L$ and $Z$. In Sect. 4.4, we discuss how to include the observed rotating longitudunal asymmetry into the model, and explore various ways to do so. These results are then synthesized into a final density model in Sect. 5 . 


\subsection{Extension of plasma disc}

Previous studies of the plasma disc region reported that the electron density distribution varies as $n_{0} \exp \left(-(Z / H)^{2}\right)$ (e.g., Hill and Michel, 1976). Although the amount of data points near Saturn in this study is restricted, the data set shows a reasonable trend which is close to $n_{0} \exp \left(-(Z / H)^{2}\right)$ inside $L=15$. Therefore, we identified the disc-like highdensity region at $L<15$ as the extension of plasma disc. The ring current has been observed in this region (Dougherty et al., 2005; Krimigis et al., 2005; Krupp et al., 2005), which extends from 8-14 $R_{S}$ in the equatorial region (Connerney et al., 1981). Recent observations showed that the boundary of the ring current region could extend further than in the Connerney model (Krimigis et al., 2007; Sergis et al., 2007).

We found that the plasma scale height $(H)$ increased with increasing L. This is consistent with the results of Persoon et al. (2006) who used densities obtained from $f_{U H}$ at $5<L<$ 9 , though the increase we see in the outer magnetosphere is slower than the relation found by Persoon et al. (2006) for data inside $L=9\left(H=0.047 L^{1.8}\right)$. Since $H$ can be defined by $H^{2}=2 K T_{i} / 3 m_{i} \Omega^{2}$, where $K T_{i}$ is the ion thermal energy, $m_{i}$ is the ion mass and $\Omega$ is the angular velocity of planetary rotation (Hill and Michel, 1976), the slower increase in scale height outside $L=9$ can correspond to a slower increase in ion temperature, a slower decrease in ion mass, and/or a decrease of the rotation speed. It is known that the rotation speed starts to decrease beyond $5 R_{S}$ (Saur et al., 2004), which is quite reasonable when we go outward in the magnetosphere. As shown by our results, the density $Z$ dependence beyond $L>$ 15 is clearly different from the distribution in the plasma disc region and $H$ has to decrease somewhere between $L=7$ to 15 .

It is also found that the values of $H$ overall seem to be larger on the dayside. This could be an indication of the magnetosphere compression by the solar wind as suggested by Krimigis et al. (2007).

\subsection{Magnetodisc}

Beyond $L=15$, the density shows a disc-like distribution, decreasing with increasing distances from Saturn as well as from the equatorial plane. This implies that the ions are still being affected by the centrifugal force, tending to confine the plasma to the equator region. This is consistent with the idea suggested by Arridge et al. (2007) that Saturn's magnetosphere forms a magnetodisc due to the rapid rotation of the planet as previously discovered in the Jovian magnetosphere (Gledhill, 1967). Following this idea we identify the region beyond $L=15$ as the magnetodisc. Recently Arridge et al. (2008) showed that the magnetic field of Saturn is distorted into a disc-like shape beyond $16 R_{S}$, and the electron density distribution observed here is consistent with that result.

The density in the magnetodisc shows a clear longitudinal asymmetry. Two sectors, the low-density and the enhanced- density sector, appear periodically in the Cassini data correlated with the SKR longitude system. Similar signatures have been found in several other Cassini data sets. Krupp et al. (2005) compared the high-energy particle (MIMI) and magnetic field data onboard Cassini, identified the lobe as a region of less intense high-energy particles and tail-like magnetic field, and the plasmasheet region as a region with intense high-energy particles and stretched dipole field line. These two regions can be related to the enhanced-density and the low-density sectors found in this study. The bottom panel of Fig. 2 shows the magnetic field data during orbit 20 . Comparing the electron density (top) and the magnetic field, we can find that the low-density sector and the enhanceddensity sectors correlate with specific magnetic field signatures. Most of the time Cassini observed the low-density sector where the $B_{r}$ and $B_{\phi}$ components are dominant, the tail-like magnetic field. In the enhanced-density sector, we find a sharp decrease of $\left|B_{r}\right|$ and $\left|B_{\phi}\right|$. Simultaneously $\left|B_{Z}\right|$ increases and becomes the dominant component, indicating a more dipole-like magnetic field. Following the identification by Krupp et al. (2005), we find the enhanced-density sector is on closed field lines with dipole like magnetic field, while the low-density sector is found on field lines with tail-like magnetic field. Note that the tail-like magnetic field signature is also consistent with the magnetodisc signature shown by Arridge et al. (2008). A longitudinally asymmetric plasma distribution, corotating with the planet, was also observed clearly for $20-50 \mathrm{keV}$ particles by Krimigis et al. (2007) using energetic neutral atoms as tracers. Further inward, Gurnett et al. (2007) found such a structure in the plasma density inside $10 R_{S}$. Our data further show that the longitudinal asymmetry applies to the plasma density to distance out to at least $30 R_{S}$.

At the equator, the density oscillates between the lower (linear to $L^{-6}$ ) and the upper (linear to $L^{-3}$ ) limit lines because of the rotating longitudinal asymmetry. As a result, the averaged density trend matches the density model by Persoon et al. (2005). Assuming a dipole magnetic field Persoon et al. (2006) suggested an equatorial density distribution model in the inner magnetosphere inside $9 R_{S}$ based on the conservation of the magnetic flux, $\mathrm{BA}=$ constant, giving

$n_{e}=N / A(2 H)$.

Since the dipole magnetic field follows $R^{-3}$, leading to $A \propto$ $R^{3}$, and $H$ is proportional to $R$ as reported by Moncuquet et al. (2005), the density depends on $R^{-4}$ (equivalent to $L^{-4}$ in the equatorial plane). This relation matches the distribution at $L<9$ by Persoon et al. (2006) and the averaged distribution of the density in this study. However, as discussed before, we expect that the magnetic field in the low-density sector is tail-like and in this case the formula should be modified. The magnetic field of the planetary wind is (Hill et al., 1974) 
$B(L)=B_{0}\left(\frac{1}{R^{3}}+\frac{1}{R_{A}^{2} R}\right)$

where $R_{A}$ is called Alfvén radius, the distance where the magnetic field starts to form a planetary wind due to the centrifugal force. As a result, in the distant magnetosphere the magnetic field is nearly proportional to $R^{-1}$. In the distant tail we found that the lower limit of the density is proportional to $L^{-1.5}$. Combining this magnetic field with the density dependence in the distant magnetosphere in the low-density sector implies $H \propto R^{-0.5}$. This is consistent with the nightside $Z$ distribution, which we found to vary as $\exp \left(-Z^{0.5}\right)$. We also found that the $Z$ distribution inside $L=25$ is slightly different from the distribution beyond $L=25$. This might correspond to the height scale changing due to the magnetic field configuration. On the tail-like field configuration, the centrifugal force becomes parallel to the magnetic field, and the height scale should be modified. Further studies are needed to establish the density distribution under a effect of the centrifugal force parallel to the magnetic field.

\subsection{Lobe}

Also beyond $L=15$, the density increases toward the equatorial plane. On the other hand, in the high latitude region $\left(Z>10 R_{S}\right)$ the density is approximately constant. This implies that the effect of the centrifugal force is significant mostly for $Z<10 R_{S}$ and becomes weaker at high latitude. This high latitude region we identify as the lobe region. Note that the periodic density variation has been observed not only near the equatorial plane but also in the high latitude region. Thus the longitudinal asymmetry of the density in Saturn's magnetosphere extends also into the lobes. The alternative to explain this variability as a repetitive crossing of the spacecraft between the lobe and the mantle regions associated with the planetary rotational dynamics of the plasma sheet we consider less likely, as the behaviour appears similarly for all $Z$.

\subsection{The longitudinal variation of the electron density}

In the sections above, we have discussed the density characteristics in L-values and the latitudes $(Z)$ region. In addition to those characteristics, the longitudinal variation of the electron density was obtained in this study. Here, we discuss the longitudinal characteristics and investigate some quantitative models for describing it. Although we observed longitudinal density variations both on the dayside and the nightside, the amount of data on the dayside is limited since we avoided the data when the spacecraft repetitively get into the magnetopause. Therefore here we will base our discussion on the data from the nightside. The density model on the dayside must be established elsewhere taking into account the plasmapause location and a more complete data set.

\subsubsection{Density model with a longitudinal asymmetry}

We will first study a model where the longitudinal (rotating) asymmetry is regarded as the sole cause of the observed quasi-periodic density variation. Figure 9a shows an electron density model for the magnetodisc region $(L>15)$. The model is three-dimensional defined by $L$ (L-value), $\phi$ (SKR longitude in radian), and $Z$ (the distance form the equatorial plane in $R_{S}$ ). The panels (a) and (b) show the electron density at $\phi=0$ and $\phi=\pi$, respectively. For given $(L, \phi, Z)$ the electron density $N_{e}$ is defined as:

$N_{e}(L, \varphi, Z)=\left\{\begin{array}{l}N_{0}(L, \varphi) \exp \left\{-Z^{0.6}\right\}(\mathrm{Z}>0) \\ N_{0}(L, \varphi) \exp \left\{-Z^{0.8}\right\}(\mathrm{Z}<0)\end{array}\right.$

where $N_{0}(L, \phi)$ is the density in the equatorial plane defined as

$$
\begin{aligned}
N_{0}(L, \varphi) & =\left\{N_{\text {High }}(L)-N_{\text {Low }}(L)\right\} \\
& \left\{\exp \left(-\frac{\left[\varphi-\varphi_{0}\right]^{2}}{\alpha}\right)+\exp \left(-\frac{\left[2 \pi-\varphi+\varphi_{0}\right]^{2}}{\alpha}\right)\right\} \\
& +N_{\text {Low }}(L) \\
N_{\text {High }}(L) 7= & 2 \times 10^{4} L^{-3} \\
N_{\text {Low }}(L) & =6 \times 10^{6} L^{-7}+6 \times 10^{0} \cdot L^{-1.5} \\
& {[0 \leq \varphi \leq 2 \pi] }
\end{aligned}
$$

$\phi_{0}$ and $\alpha$ are the centre longitude and the degree of localization of the high-density region, and set to be $\phi_{0}=0$ and $\alpha=1$, respectively, in this study.

The functional form of the longitudinal dependence is discussed below and shown in panel (d). In the lobe region at $|Z|>15 R_{S}, N_{\text {High }}$ and $N_{\text {Low }}$ are set to constant values,

$N_{\text {High,lobe }}=5 \times 10^{-4} \mathrm{~cm}^{-3}$

$N_{\text {Low, lobe }}=5 \times 10^{-3} \mathrm{~cm}^{-3}\left(|Z|>15 R_{S}\right)$

Panel (c) shows the electron densities for the orbit 38. The blue dots are the data from orbit 38 (same as shown in Fig. 3) and the black dots are the calculated model along the Cassini trajectory during the orbit 38 (blue lines in panels a and b). The model reasonably reproduces the data: the density decreases with increasing $Z$ close to as observed, and the variations agree very well also in phase.

To represent the longitudinal dependence in Eq. (5), a double Gaussian was chosen because we found that enhanceddensity sector appears longitudinally localised region in the data and fits better with a Gaussian rather than a sinusoidal function $\left(\sin ^{2}(\phi / 2)\right)$. A trigonometric sum, which also gives similar shape, can also applied for the longitudinal dependence, however, we deem the form used above potentially more useful, as it allows direct interpretation in terms of a typical longitudinal width $\alpha$ (in this case 1 radian) of the region of enhanced density and a clear phase $\phi_{0}$ with respect to the zero longitude line (in this case set to zero). With a large statistical material, these parameters can be fitted to the data. The present data set is not sufficiently large to merit such a fit, and we here use the values given for $\alpha$ and $\phi_{0}$ simply on the grounds that they sufficiently well represent the data. 

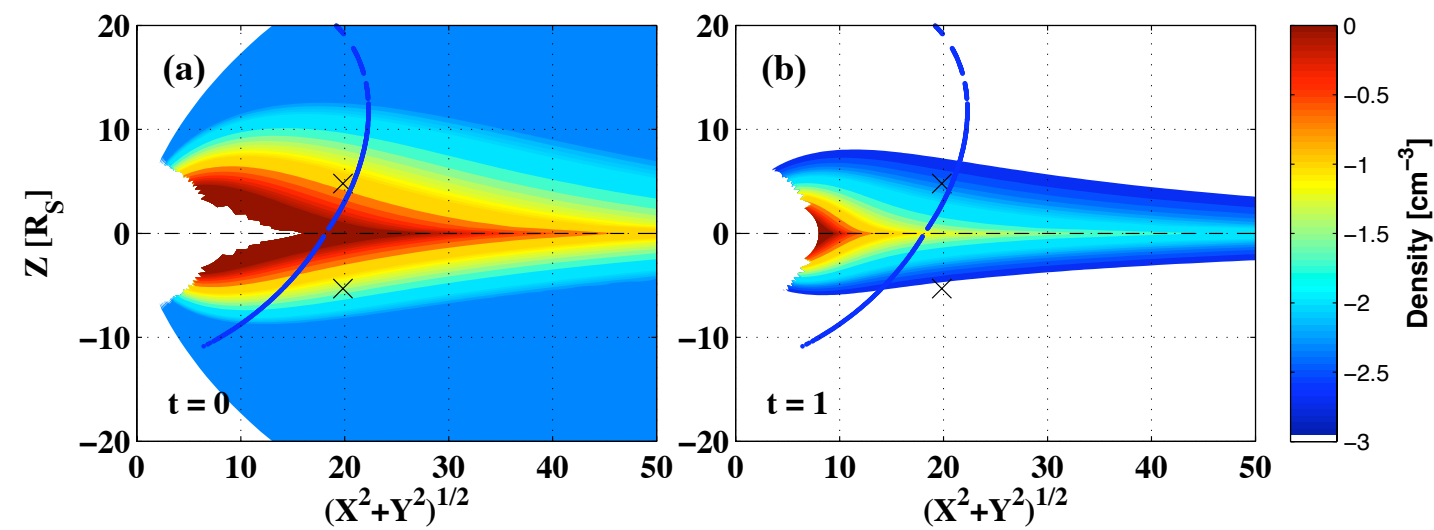

Density of the CS center at $X=19.8$
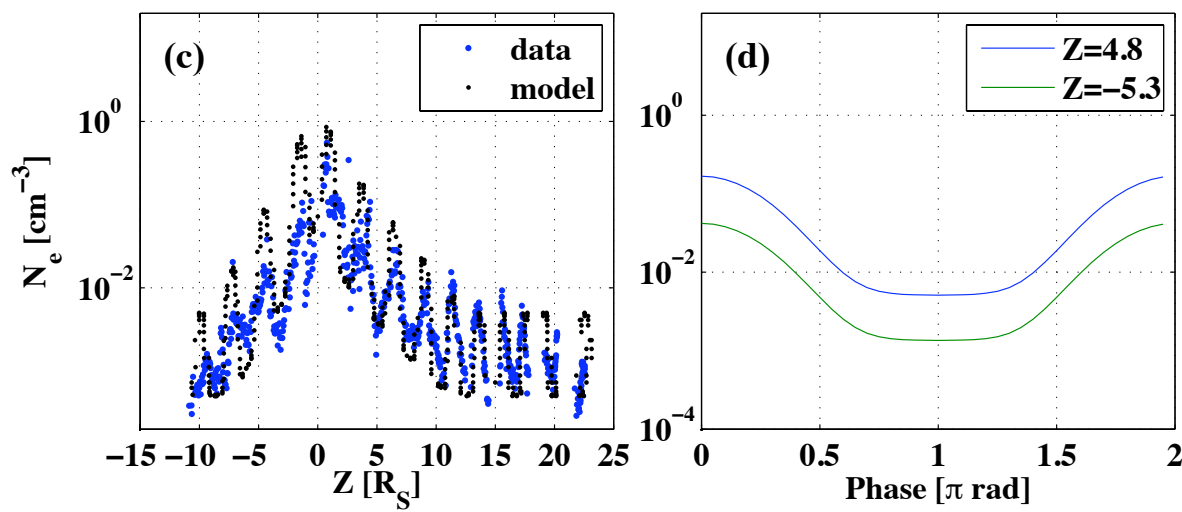

CS center position at $X=19.8$

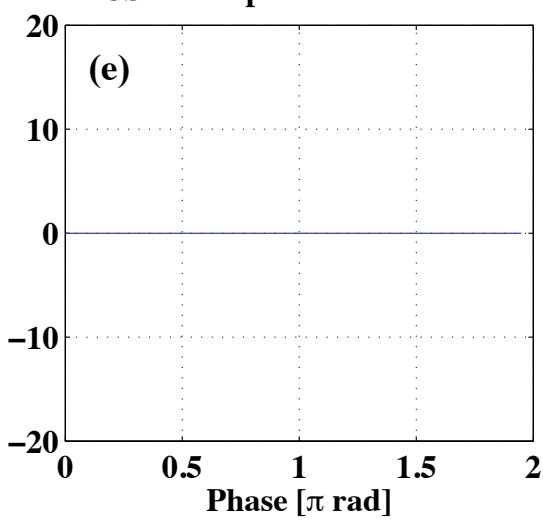

Fig. 9a. A magnetodisc density model with longitudinal asymmetry. (a) Model density in the high density sector (SKR longitude $\phi=0^{\circ}$ ). Blue line is the trajectory of Cassini for orbit 38, where Cassini was located in the nightside (MLT=23:00-03:00 h) magnetosphere. (b) Model density in the low-density sector $(\phi=\pi)$. (c) Comparison of model (blue) to data from orbit 38 (black). (d) The model density variation with longitude at $(X, Z) \approx\left(20 R_{S}, 5 R_{S}\right)$ and $\left(20 R_{S},-5 R_{S}\right)$. These locations are marked by crosses in panels (a) and (b). (e) displacement of the plasma disc centre from the magnetic equatorial plane, shown at $X \approx 20 R_{S}$ : in this model, there is no such displacement. See Sect. 4.4 for a detailed description of the model.

\subsubsection{Density model with a longitudinal asymmetry of the magnetodisc location}

As an alternative possibility, we also consider an explanation of the rotating asymmetry in terms of a latitudinal change of the magnetodisc with respect to the spacecraft. While the intrinsic dipole is very close to the rotation axis (Dougherty et al., 2005), field-aligned currents in the inner magnetosphere have been suggested to cause a magnetic field, which in the outer magnetosphere would be indistinguishable from a tilted dipole (Southwood and Kivelson, 2007). If the magnetodisc moves in $Z$, the density enhancement will be observed when the spacecraft enters the magnetodisc region.

Figure $9 \mathrm{~b}$ shows the electron density model with a periodically flapping magnetodisc. For this case, the electron density is longitudinally uniform. Therefore the longitudinal asymmetry defined in Eq. (5) is replaced by

$$
N_{0}(L, \varphi)=N_{\mathrm{High}}(L)
$$

Instead here, we used the model of the location of the magnetodisc centre with a longitudinal asymmetry, and Eq. (4) is replaced by

$$
\begin{aligned}
& N_{e}=N_{0}(L, \varphi) \exp \left\{-\left|Z-Z_{C S}\right|^{0.6}\right\}\left(Z \geq Z_{C S}\right) \\
& N_{0}(L, \varphi) \exp \left\{-\left|Z-Z_{C S}\right|^{0.8}\right\}\left(Z<Z_{C S}\right)
\end{aligned}
$$

where $Z_{C S}$ is the magnetodisc centre defined as:

$Z_{C S}=\left\{X-R_{h} \tanh \left(X / R_{h}\right)\right\} \exp \left\{-\frac{\left(\pi-\varphi+\varphi_{0}\right)^{2}}{\alpha}\right\}$

We have here used the bowl shaped current sheet model (Arridge et al., 2008) to get the highest latitude of the current sheet. $R_{h}$ is the hinging distance, which is the characteristic distance where the current sheet warping starts, and here chosen to be $20 R_{S}$. As a result, the magnetodisc centre is set at the equatorial plane at $\phi=0$, and the disc has a bowl shape at $\phi=\pi$. The latitudinal $(Z)$ variation of the magnetodisc centre is shown in panel (e). In the present model the 

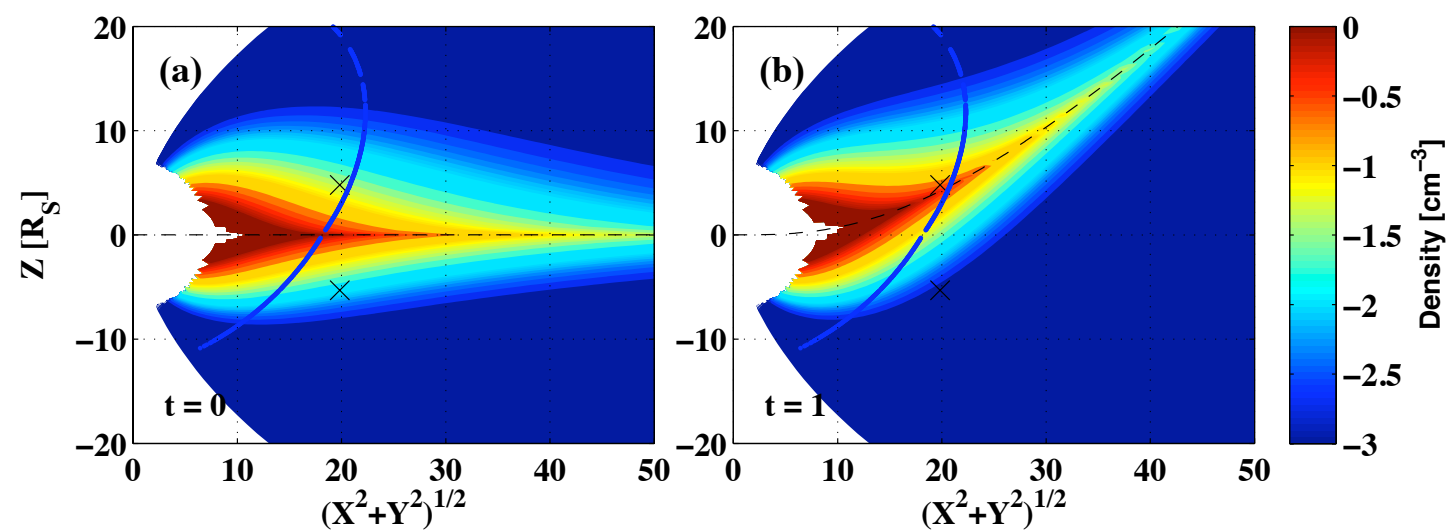

Density of the CS center at $X=19.8$
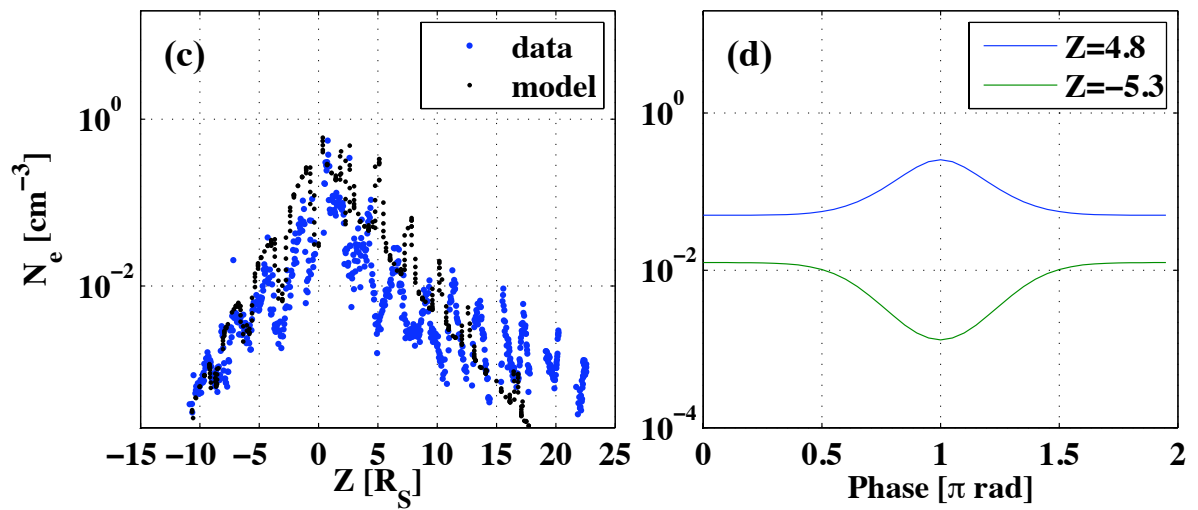

CS center position at $X=19.8$

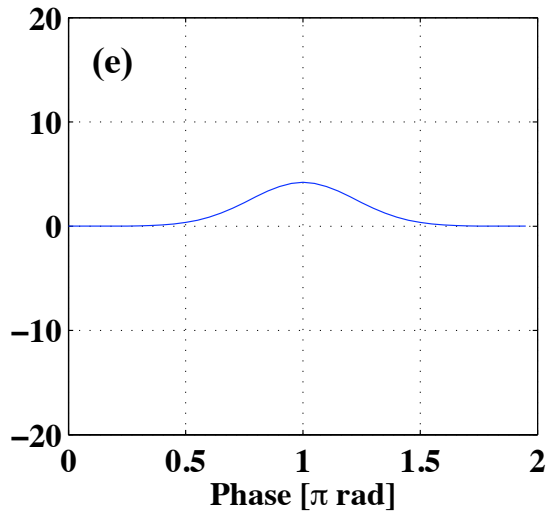

Fig. 9b. A magnetodisc density model with no other longitudinal asymmetry than a varying current sheet displacement. The plot description is same as Fig. 9a, but the model has longitudinal asymmetry for magnetodisc centre. A detailed description of this model is given in Sect. 4.4 .

magnetodisc centre at $X \approx 20 R_{S}$ moves between $Z=0$ and $4 R_{S}$.

The black dots in panel (c) show the density calculated by this model for orbit 38 . The model density reproduces the general characteristics, i.e. a decreasing trend with $|Z|$ and the periodic variation. Comparing the model density with the data from orbit 38 (blue dots), the density variation fits rather well in the Southern Hemisphere $(Z<0)$. However, there is a significant shift in $Z$ between the model and the data in the Northern Hemisphere $(Z>0)$. This is because in the latitudinal flapping magnetodisc model the density varies with the relative distance of the magnetodisc centre from the spacecraft, so the density variation is out of phase between the Northern and Southern Hemisphere. Panel (d) shows clearly the difference of the longitudinal variability in the northern $(Z \approx 5$, blue line) and in the Southern $(Z \approx-5$, green line) Hemisphere that is out of phase.

The density variation could possibly be shifted also due to the local time variation of the spacecraft orbit if the magnetodisc has a spiral structure as reported by Carbary et al. (2007). However, using the phase delay of the spiral from Carbary et al. (2007), 2.7-4.7\%/Rs, and the variations of the magnetic local time $(0-4 \mathrm{~h})$ and the distance from Saturn of Cassini $\left(27-17 R_{S}\right)$ during the orbit 38 , the phase shift can be only $13-33^{\circ}$, while the phase shift due to the magnetodisc flapping would be $180^{\circ}$.

Comparing the data from a single orbit and the density calculated by the models above, we can see that the periodic variation of the electron density can be explained by a longitudinally asymmetric structure of Saturn's magnetosphere for the orbit 38. Also as a statistical result, if the periodic variation is mainly due to the magnetodisc flapping motion, we could obtain the average centre and the thickness of the magnetodisc from the $Z$ distribution. However, we couldn't find any significant density maximum above the equatorial region beyond $L=25 R_{S}$. Also, assuming a flapping magnetodisc gives out of phase densities in the North and South Hemisphere, which contradicts to what we observe. Therefore we regard the quasi-periodic density variation as mainly due to a longitudinal asymmetry of the magnetodisc region, in effect a slab at constant longitude rotating with the planet like the cam in the model of Espinosa et al. (2003), and regard the model presented in Sect. 4.4.2 to better represent reality. We will now go on to refine that model. 


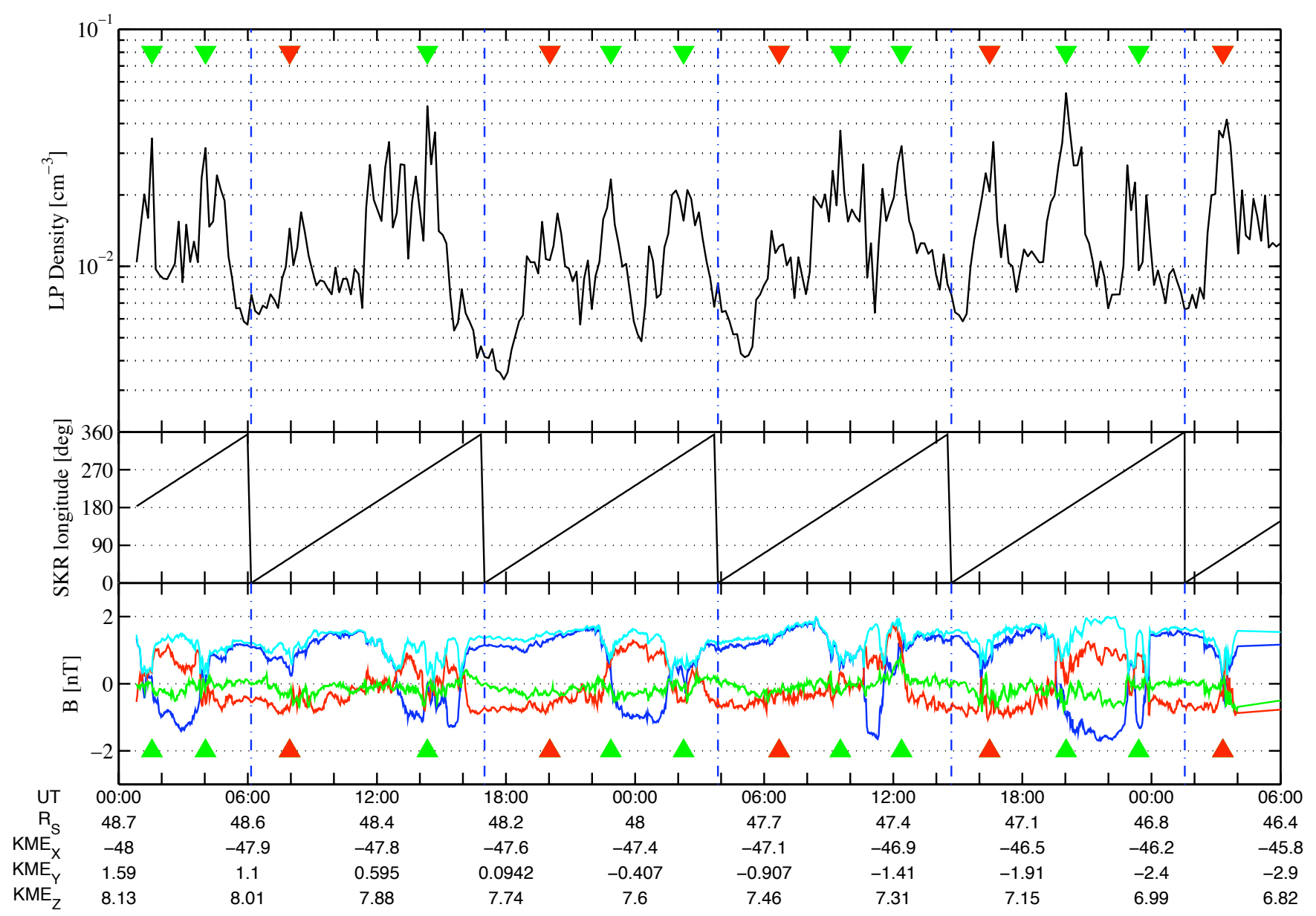

Fig. 10a. The electron densities obtained from 6 to 8 August 2006, when Cassini was located around $Z \approx 8 R_{S}$ (the time interval is included in orbit 027 ). Similar to the format of Fig. 2: from the top, the electron density by LP proxy method, the SKR longitudes, and the magnetic field components obtained by MAG. The magnetic field components are in the same colours as in Fig. 2. The triangles in the top and bottom panels mark the times when enhanced-electron densities have been observed. The green triangles are associated with magnetodisc crossings as indicated by the polarization of $\mathrm{Br}$ and $B \phi$.

\subsubsection{Density model with the longitudinal asymmetry of the density and the location of the magnetodisc}

As we see in Fig. 8b, in most cases the electron density distribution on the nightside quite well follows $\exp \left(-|Z|^{0.5}\right)$. However, sometimes the electron densities are much larger than the averaged values. Especially at $Z \approx 8 R_{S}$ on the nightside, we find densities higher than expected around $Z \approx 8 R_{S}$. This turns out to be because the spacecraft entered the enhanced density region many times during one SKR period. Figure 10a shows an example of electron density data obtained at $Z \approx 8 R_{S}$. One can find many density enhancements (indicated with the triangles at the top of the panel) in one SKR cycle. They are often found three times in a SKR period, which is more frequent than the usual case such as we see for orbit 20 (Fig. 2). The bottom panel of Fig. 10a shows the magnetic field data. Comparing the density peaks to the radial component of the magnetic field, $B_{r}$ (blue), the den- sity enhancements are often (green marks) associated with a direction change of $B_{r}$. Here they are also associated with the direction change of the azimuthal component, $B_{\phi}$ (red).

We previously found that the displacement of the magnetodisc above the equatorial plane is insignificant in the single events as well as in our statistical study. A plausible explanation of these signatures in this particular event is that the spacecraft encountered the centre of the magnetodisc away from the equatorial plane, just like we considered in Fig. $9 \mathrm{~b}$. If the magnetodisc is flapping latitudinally, the spacecraft crosses the centre twice, from the Northern Hemisphere to the Southern Hemisphere and back to the Northern Hemisphere again. Similar magnetic field signatures have been observed in Jupiter's magnetosphere, in which the magnetic dipole is tilted from the rotation axis by about $9.5^{\circ}$ (Khurana and Schwarzl, 2005). Two of the three density enhancements (the ones marked with green) during one SKR period which associated to $B_{r}=0$ crossing can be explained by the current 

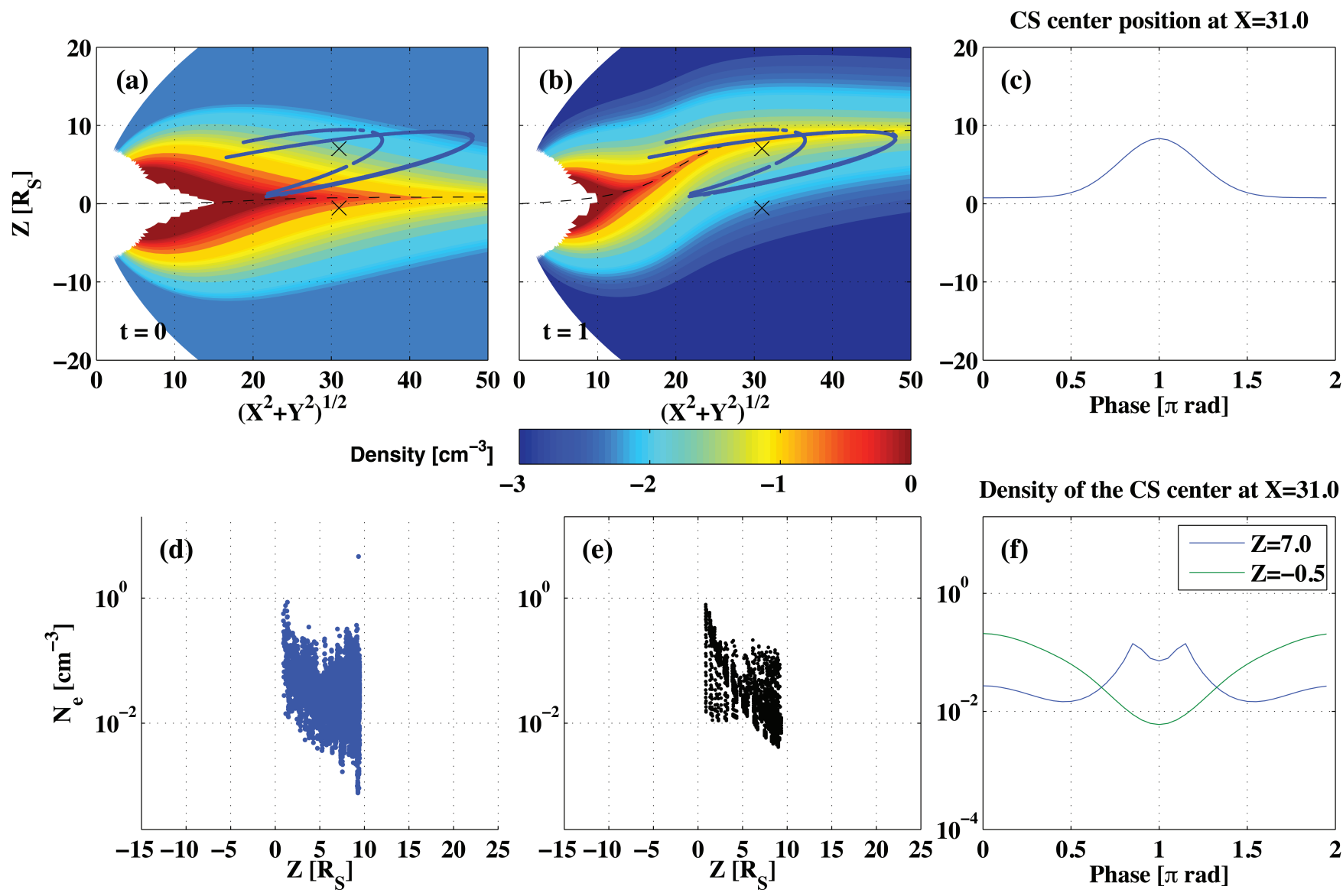

Density of the CS center at $X=31.0$
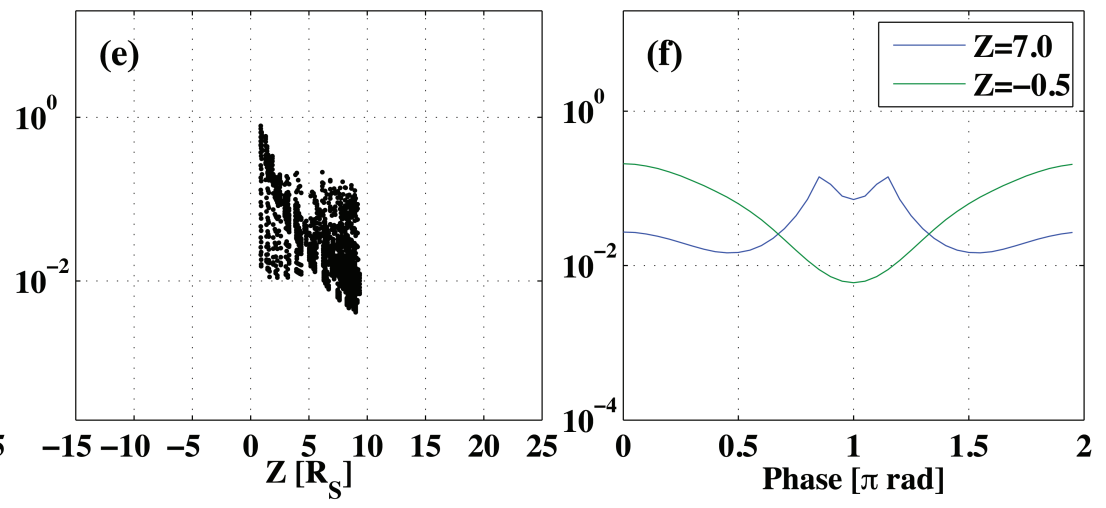

Fig. 10b. A magnetosphere density model with longitudinal asymmetry and flapping motion. (a) and (b) The model density in the highdensity sector (a) and low-density sector (b). Blue lines are the Cassini's trajectory during the orbits $27-29$ (4 August-9 September 2006). (c) Centre of the magnetodisc from the magnetic equatorial plane at $X \approx 30 R_{S}$. (d) the LP density data obtained during the orbits $27-$ 29. (e) The model density along the trajectory of the orbit 27-29 (blue lines in panels a and b). (e) The model density variations at $(X, Z) \approx\left(30 R_{S}, 7 R_{S}\right)$ and $\left(30 R_{S}, 0 R_{S}\right)$. These locations are marked by crosses in panels (a) and (b). See Sect. 4.5 for a detailed model description.

sheet crossings of the spacecraft when the magnetodisc centre was located well above the equatorial plane.

On the other hand, we still observed another group of density enhancements in the same event (red marks). They are observed at the opposite SKR longitude compared to the green group density enhancements, and not associated with the zero crossing of $B_{r}$ and $B_{\phi}$.

Khurana et al. (2009) suggested a mechanism of the magnetodisc flapping as responsible for the periodic signatures of Saturn's magnetosphere. They suggested that the solar wind lifts longitudinally asymmetric Saturn's magnetosphere, generating an asymmetric tilt in the current sheet of Saturn. In this model, the lower density sector would be more easily pushed above the equatorial plane while the heavier sector is still confined near the equatorial plane due to a strong centrifugal force effect. Following this idea, we construct a density model with a longitudinally asymmetric magnetodisc, resulting in an apparently flapping motion of the magnetodisc as the planet rotates. The model is displayed in Fig. 10b. For this model, functions are combinations of the longitudinal density asymmetry model (Sect. 4.4.1) and the flapping disc mode (Sect. 4.4.2) as follows:

$N_{e}(L, \varphi, Z)=N_{0}(L, \varphi) \exp \left\{-\left|Z-Z_{C S}\right|^{0.6}\right\}$

where the density in the magnetodisc centre $N_{0}(L, \phi)$ is defined as:

$$
\begin{aligned}
& N_{0}(L, \varphi)=\left\{N_{\text {High }}(L)-7 \cdot N_{\text {Low }}(L)\right\} \\
& {\left[\exp \left\{-\frac{\left[\varphi-\varphi_{0}\right]^{2}}{\alpha}\right\}+\exp \left\{-\frac{\left[2 \pi-\varphi+\varphi_{0}\right]^{2}}{\alpha}\right\}\right]} \\
& +7 \cdot N_{\text {Low }}(L) \\
& N_{\text {High }}(L)=2 \times 10^{4} L^{-3} \\
& N_{\text {Low }}(L)=6 \times 10^{6} L^{-7}+6 \times 10^{0} \cdot L^{-1.5} \\
& {[0 \leq \varphi \leq 2 \pi]}
\end{aligned}
$$


and the location of the magnetodisc centre $Z_{C S}(Z)$ is defined as:

$$
\begin{aligned}
Z_{C S}= & \left\{Z_{C S 1} \exp \left(-\frac{\left[\pi-\varphi+\varphi_{0}\right]^{2}}{\alpha}\right)-Z_{C S 0}\right\} \\
& \left\{\tanh \left(\frac{10(x-20)}{180} \pi\right)+0.4 \pi\right\}
\end{aligned}
$$

$Z_{C S 0}$ and $Z_{C S 1}$ are set 1 and 10 , respectively.

Some parameters are slightly changed from Eqs. (4-9). These parameters will be discussed later. The present model gives the densities shown in panels (a) and (b). In the enhanced-density sector at $\phi=0$ (shown in panel a), the disc centre is located around the equatorial plane. The lowdensity sector appears at $\phi=\pi$ (panel b), where the disc centre is displaced at $Z=8 R_{S}$. Panel (c) shows the latitudinal distribution of the magnetodisc centre at $X=31 R_{S}$. The centre reaches to maximum in $Z$ at $\phi=\pi$ which corresponds to panel (b). These signatures are consistent with the magnetodisc dynamics model by Khurana et al. (2009). The longitudinal distribution of the densities at $X=31 R_{S}$ are shown in panel (f). Near the equatorial plane (green line, represents $Z \approx 0$ ), the density has one maximum at $\phi=0^{\circ}$. On the other hand, at $Z=7 R_{S}$ (blue line), the density has three maxima: Two maxima appear near $\phi=\pi$. These occur when the magnetodisc centre crosses $Z=7 R_{S}$. Another maximum appears at $\phi=0^{\circ}$ in the enhanced-density sector. These three density maxima are similar to the density periodicity observed around $Z=8 R_{S}$ in Fig. 10a.

Panel (d) shows the electron densities observed during August and October in 2006 (orbit 27, 28, and 29), when Cassini was located in the nightside with $Z=0-8 R_{S}$. These specific trajectories are marked with light blue lines in Fig. 4 and also marked in Fig. 5 as well. Panel (e) shows the model densities calculated along the Cassini trajectories during the orbit 27-29 (blue lines in panels a and b). In the observed data (panel d) one can find two density maximum regions: one is around the equatorial plane, and another is around $Z=8 R_{S}$. Two density maxima can be also found in the model at similar $Z$ values (panel e). By comparing the data and model densities, we find that the density maximum at the equatorial plane can be obtained in the enhanced-density sector, while the other two density maxima at $Z=8 R_{S}$ can be obtained when the current sheet is displaced in large $Z$. These characteristics are reproduced well in the model.

To describe the shape of the magnetodisc (Eq. 12) uses tangential function rather than the bowl shape. This is because that the signature of the current sheet crossing (density peaks associated with $B_{r}$ change) has been observed near $Z=8 R_{S}$ in a large range in $X$ at $20 R_{S}<R<40 R_{S}$.

We find here that the present density model with longitudinal asymmetry and latitudinal flapping motion of the magnetodisc reasonably explain the data obtained during the orbit 27-29. However, the most of the other orbits can still be explained only with the longitudinally asymmetric model as described in Sect. 4.4.1. This implies that the plasma condition in Saturn's magnetosphere was different from the other time intervals during this time interval. André et al. (2008) reported that there are evidences that the $Z$ location of the magnetic equatorial plane moved dramatically northward during the time interval of orbits 26 and 27, and they were also associated with a strong of SKR emissions. They suggest that the reconfiguration of the magnetosphere has been triggered by a solar wind disturbance. It is also known that the SKR longitude system had a large phase shift around the orbit 26-27, which can be explained by a large disturbance in Saturn's magnetosphere (Kurth et al., 2007). We suggest that the flapping motion of the magnetodisc becomes large as suggested by Khurana et al. (2009) in the midnight when a strong solar wind condition. In Fig. 4, is shown that the Cassini's position during the orbit 27-29 was near the midnight, where the tilt effect of the current sheet by the solar wind can be most effective. Perhaps the combination of the temporal solar wind variation and the special orbital location during this time interval made it possible to see the flapping motion of the magnetodisc.

Note that, in this model, the density of the low-density sector is multiplied by 7 in Eq. (11) in order to explain a large density enhancement around $Z=8 R_{S}$. The plasma condition in the Saturnian magnetosphere itself could also be different from the other orbit in this time interval. Therefore, the density characteristics obtained in the orbit 27-29 suggest that Saturn's magnetosphere can be dynamic due to both external (solar wind) as well as internal (plasma source) conditions.

\section{An electron density model for Saturn's magnetosphere}

From the electron density characteristics in $L$ and $Z$ obtained in this study, we have constructed an electron density model for the Saturnian magnetosphere. The density calculated by this model is shown in Fig. 11. From both single event studies and the statistical analysis, we regard a corotating longitudinal asymmetry as the cause of the periodic variability observed in the Cassini LP data. A flapping motion of the magnetodisc can co-exist with this rotating asymmetry if the amplitude of the magnetodisc latitudinal displacement is not too large. Therefore, we have accounted for a small displacement of the magnetodisc centre. However, the latitudinal variation of the magnetodisc must be quite small, as a large magnetodisc displacement would result in extremely low densities near the equatorial region, which we do not find in our observation. As a result, our model of the electron density in the magnetosphere has a longitudinally asymmetric structure where the density is high and centred around the geographic equatorial plane on one side of the SKR longitude system (panel a), while the density is low and the centre is displaced slightly northward on the other side (panel $b$ ). The magnetodisc centre at $X=20 R_{S}$ is placed at $Z \approx 1.3 R_{S}$ 

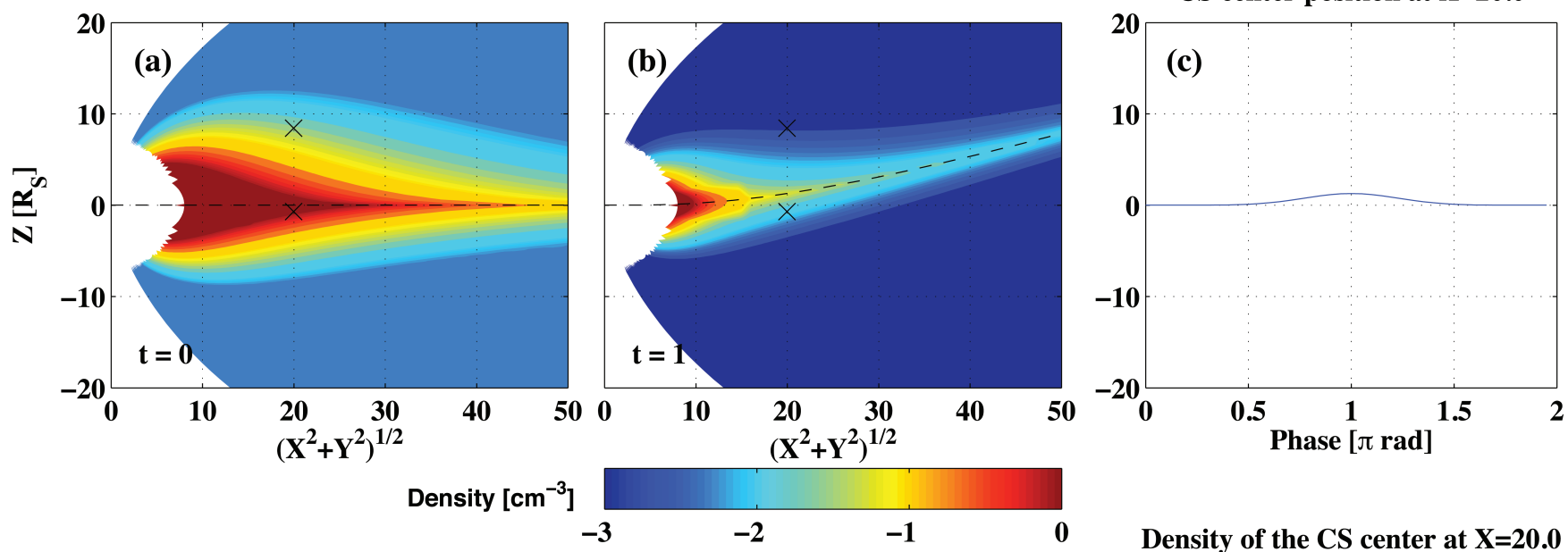

Density of the CS center at $\mathrm{X}=\mathbf{2 0 . 0}$
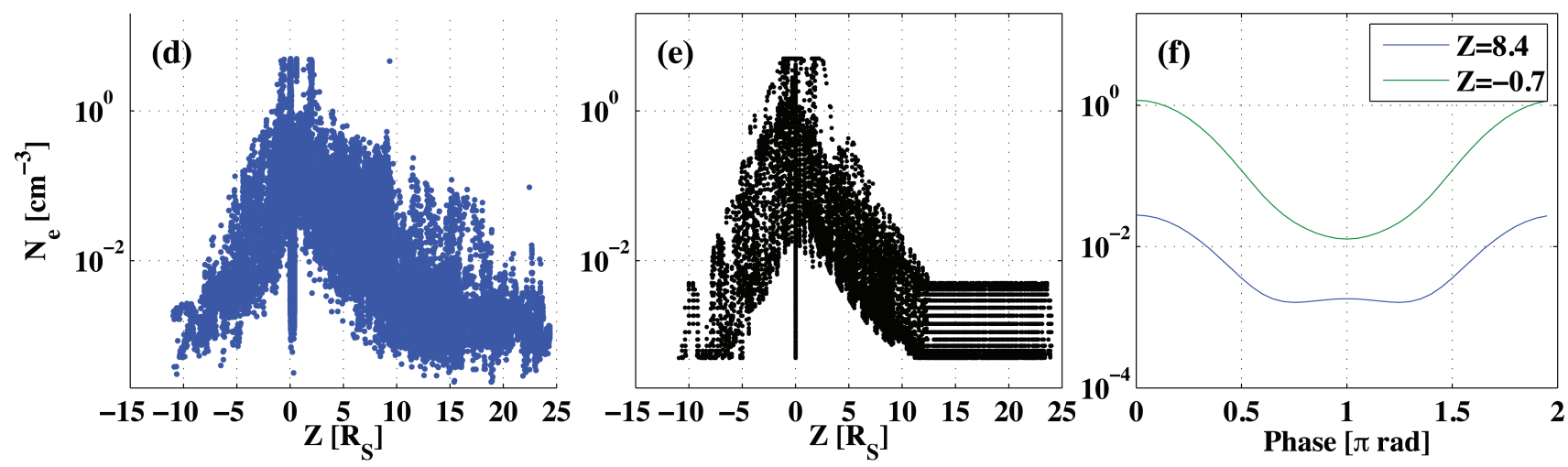

Fig. 11. Our density model for Saturns magnetosphere. (a) Density model for the high-density sector $\left(\phi=0^{\circ}\right)$. (b) Density for the low-density sector $(\phi=\pi)$. (c) The longitudinal variation of the magnetodisc centre position at $X=20 R_{S}$. The current sheet is set slightly above the equatorial plane at long $=180^{\circ}$. (d) Scatter plot of the observed plasma density on the nightside. (e) Scatter plot of the density calculated from our model using the Cassini trajectories corresponding to the data in panel (d). (f) The longitudinal variation of the density in this model at $X=20 R_{S}$, in the Northern Hemisphere $\left(Z \approx 8 R_{S}\right)$ and near the equatorial plane $(Z \approx 0)$. These locations are marked by crosses in panels $\mathrm{a}$ and $\mathrm{b}$. The model densities (panel e) and the observation (panel d) overall agree very well with respect to that the density maximum is located around the equatorial region and decrease with $|Z|$, the density is constant against $Z$ at $Z>10$, and the density variation is large in all $Z$. There are some disagreements between the model and the data around $8 R_{S}$, which can be due to a sadden solar wind compression occurred during the time interval of Rev 27-29 (See the text in Sect. 4.4.3 for details).

at $\phi=\pi$ (panel c). Our final electron density model, well fitting what was typically observed in this study, is therefore described as follows:

Inside $L=15$ is the extension of plasma disc region where the density is defined as:

$$
\begin{aligned}
& N_{e}=N_{0}(L, \varphi) \cdot \exp \left(-(Z / H(L))^{2}\right)(L<15) \\
& H(L)=0.58 L^{0.39}
\end{aligned}
$$

Beyond $L=15$ is the magnetodisc region, where the density is given by

$$
\begin{aligned}
& N_{e}=N_{0}(L, \varphi) \exp \left\{-\left|Z-Z_{C S}\right|^{0.6}\right\}\left(Z \geq Z_{C S}\right) \\
& N_{0}(L, \varphi) \exp \left\{-\left|Z-Z_{C S}\right|^{0.8}\right\}\left(Z<Z_{C S}\right)
\end{aligned}
$$

where $Z_{C S}$ is the magnetodisc centre defined as:

$$
\begin{gathered}
Z_{C S}=0.3 \cdot\left(X-R_{h} \tanh \left(X / R_{h}\right)\right. \\
R_{h}=20 R_{S}
\end{gathered}
$$

For all regions in the magnetosphere, the density has a longitudinal asymmetry. The density function in the equatorial plane is defined as:

$$
\begin{aligned}
N_{0}(L, \varphi)= & \left\{N_{\text {High }}(L)-N_{\text {Low }}(L)\right\}\left[\exp \left\{-\frac{\left[\varphi-\varphi_{0}\right]^{2}}{\alpha}\right\}\right. \\
& \left.+\exp \left\{-\frac{\left[2 \pi-\varphi+\varphi_{0}\right]^{2}}{\alpha}\right\}\right]+N_{\text {Low }}(L) \\
{[0 \leq \varphi \leq 2 \pi] } &
\end{aligned}
$$

where the densities in the equatorial regions in the magnetodisc are defined as:

$$
\begin{aligned}
& N_{\text {High }}(L)=2 \cdot 10^{4} L^{-3} \\
& N_{\text {Low }}(L)=6 \cdot 10^{6} L^{-7}+6 \cdot 10^{0} \cdot L^{-1.5}
\end{aligned}
$$


The centre longitude $\left(\phi_{0}\right)$ and the degree of localization $(\alpha)$ of the high-density region are set to $\phi_{0}=0$ and $\alpha=1$, respectively.

The lobe region is at $|Z|>15 R_{S}$ and the densities have a constant distribution defined as:

$$
\begin{aligned}
& N_{\text {High,lobe }}=5 \times 10^{-4} \mathrm{~cm}^{-3} \\
& N_{\text {Low,lobe }}=5 \times 10^{-3} \mathrm{~cm}^{-3}\left(|Z|>15 R_{S}\right)
\end{aligned}
$$

Panel (d) shows the density characteristics in $Z$ obtained in this study. Panel (e) shows the artificial densities obtained from the density model using the Cassini orbits same as the observation in this study. Panel (e) well reproduces the actual data, shown in panel (d), including the general $Z$ dependence with a density maximizing around the equatorial plane and decreasing with increasing $|Z|$. It also well reproduces the large variability seen in the data.

This model suggests that Saturn's magnetodisc varies in thickness and latitudinal location depending on the SKR longitude under the control of Saturn's rotational force and the solar wind dynamic pressure. The thickness of the magnetodisc reported previously varies from $4 R_{S}$ (e.g., Dougherty et al., 2006) to $10 R_{S}$ (e.g., Sergis et al., 2009). All these studies can be consistent with our model as the magnetodisc thickness changes with the longitude (as seen in panels a and b). Also the plasmasheet can be thin and light when it is observed at the northern high latitudes and during a time of large solar wind pressure. Also, the observation at the northern high latitude tends to observe thin and lighter (low density) plasmasheet when the solar wind dynamic pressure is large. This characteristic is consistent with the idea by Khurana et al. (2009).

The data certainly show some densities even higher than the high-density sector densities we have modelled, for example in orbit 27. However, we regard these as special cases likely occur when the magnetosphere conditions have been changed by particular events in the solar wind or the plasma sources in the Saturnian magnetosphere.

\section{Summary}

In the tenuous plasma of the magnetosphere, the spacecraft potential gives a good estimate of the electron number density. In this case the floating potential $U_{\text {float }}$ of the Langmuir probe onboard the spacecraft, which is proportional to the spacecraft potential, can be used as proxy value of the ambient electron density. We have investigated the electron densities in Saturn's magnetosphere beyond $7 R_{S}$ using the floating potential $U_{\text {float }}$ derived from Cassini RPWS LP, and presented statistical distributions in $\mathrm{L}$ and $\mathrm{Z}$. From the density, we identified various regions in the magnetosphere: the extension of the plasma disc region, the magnetodisc region, and the lobe region.

The plasma disc lies inside $L \approx 15$ and has relatively high density (order of $10^{-1} \mathrm{~cm}^{-3}$ ). We have estimated the height scale $H$ of this region, and showed that $\mathrm{H}$ decreases with increasing the distance. It is also shown that $H$ on the dayside seems to be larger than the value on the nightside, which can be an indication of the plasmasheet compression by the solar wind as suggested by Krimigis et al. (2007).

The magnetodisc also extends beyond $L=15$ with a disclike shape and a density varying with the SKR longitude. The density distribution in the equatorial plane is similar for the plasma disc and the magnetodisc, but how the plasma density scales with height above the centre of the plasma disc (namely plasma scale height) changes as the distance from Saturn increases.

Above $Z>15 R_{S}$, we have the lobe region, where the electron density does not depend on $Z$. However the density varies with the Saturnian rotation in this region as well.

Based on the observed density characteristics as a function of various L-values, $Z$, and longitude obtained in this study, we presented a few density models of Saturn's nightside magnetosphere. The presented models represent a disclike structure of Saturn's magnetosphere and results in similar statistics as the observations. We also tested the models with various longitudinal variations to compare the observations. As a result, it is shown that Saturn's magnetosphere has a strong longitudinal asymmetry. However, the location of the magnetodisc centre can be occasionally displaced by various conditions in the magnetosphere and the solar wind.

Our final electron density distribution model for Saturn's magnetosphere is displayed in Fig. 11. As the model reproduces the observations well, it can be used for modelling the magnetosphere environment in future studies. Nevertheless, there are a number of limitations to our study. First, the orbital coverage of the Cassini data used in this study still has an asymmetry in local time. Second, we have made no attempt to include the impact of varying solar wind conditions on Saturn's magnetosphere. The SKR longitude dependence of the density should also be investigated further to elucidate the causes of the observed longitudinal asymmetry. We expect that investigations, based on a larger data set as the Cassini database continues to grow and thus having more complete orbit coverage, will further improve our understanding of the plasma environment in Saturn's magnetosphere.

Acknowledgements. This study is supported by a grant from the Swedish National Space Board (SNSB). The research at the University of Iowa is supported by NASA through JPL contract 1279973.

Topical Editor I. A. Daglis thanks A. Pedersen and N. Sergis for their help in evaluating this paper.

\section{References}

André, N., Persoon, A. M., Goldstein, J., Burch, J. L., Louarn, P., Lewis, G. R., Rymer, A. M., Coates, A. J., Kurth, W. S., Sittler, E. C., Thomsen, M. F., Crary, F. J., Dougherty, M. K., Gurnett, D. A., and Young, D. T.: Magnetic signatures of plasma-depleted 
flux tubes in the Saturnian inner magnetosphere, Geophys. Res. Lett., 34, L14108, doi:10.1029/2007GL030374, 2007.

André, N., Louarn, N. P., Arridge, C. S., Lamy, L., Hansen, K. C., Lewis, G. R., Jackman, C., Bunce, E. J., Burch, J. L., Coates, A. J., Cecconi, B., Cowley, S. W., Dougherty, M. K., Khurana, K. K., Russell, C. T., Sittler, E. C., and Zarka, P.: Effects of a corotating interaction region on the structure and dynamics of the Saturnian magnetosphere, The symposium for "Saturn After Cassini-Huygens", 2008.

Arridge, C. S., Russell, C. T., Khurana, K. K., Achilleos, N., André, N., Rymer, A. M., Dougherty, M. K., and Coates, A. J.: Mass of Saturn's magnetodisc: Cassini observations, Geophys. Res. Lett., 34, L09108, doi:10.1029/2006GL028921, 2007.

Arridge, C. S., Russell, C. T., Khurana, K. K., Achilleos, N., Cowley, S. W. H., Dougherty, M. K., Southwood, D. J., Bunce, E. J.: Saturn's magnetodisc current sheet, J. Geophys. Res., 113, A04214, doi:10.1029/2007JA012540, 2008.

Arridge, C. S., Khurana, K. K., Russell, C. T., Southwood, D. J., Achilleos, N., Dougherty, M. K., Coates, A. J., and Leinweber, H. K.: Warping of Saturn's magnetospheric and magnetotail current sheets, J. Geophys. Res., 113, A08217, doi:10.1029/2007JA012963, 2008.

Burch, J. L., Goldstein, J., Lewis, W. S., Young, D. T., Coates, A. J., Dougherty, M. K., and Andre, N.: Tethys and Dione as sources of outward-flowing plasma in Saturn's magnetosphere, Nature, 447, 833-835, 2007.

Carbary, J. F., Mitchell, D. G., Krimigis, S. M., and Krupp, N.: Evidence for spiral pattern in Saturn's magnetosphere using the new SKR longitudes, Geophys. Res. Lett., 34, L13105, doi:10.1029/2007GL030167, 2007.

Carbary, J. F., Mitchell, D. G., Brandt, P., Paranicas, C., and Krimigis, S. M.: ENA periodicities at Saturn, Geophys. Res. Lett., 35, L07102, doi:10.1029/2008GL033230, 2008.

Clarke, K. E., Andre, N., Andrews, D. J., Coates, A. J., Cowley, S. W. H., Dougherty, M. K., Lewis, G. R., McAndrews, H. J., Nichols, J. D., Robinson, T. R., and Wright, D. M.: Cassini observations of planetary-period oscillations of Saturn's magnetopause, Geophys. Res. Lett., 33, L23104, doi:10.1029/2006GL027821, 2006.

Connerney, J. E. P., Acuna, M. H., and Ness, N. F.: Saturn's ring current and inner magnetosphere, Nature, 292, 724-726, 1981.

Cully, C. M., Ergun, R. E., and Eriksson, A. I.: Electrostatic structure around spacecraft in tenuous plasmas, J. Geophys. Res., 112, A09211, doi:10.1029/2007JA012269, 2007.

Desch, M. D. and Kaiser M. L.: Voyager measurements of the rotation period of Saturn's magnetic field, Geophys. Res. Lett., 8, 253-256 1981.

Dougherty, M. K., Achilleos, N., Andre, N., Arridge, C. S., Balogh, A., Bertucci, C., Burton, M. E., Cowley, S. W. H., Erdos, G., Giampieri, G., Glassmeier, K. H., Khurana, K. K., Leisner, J., Neubauer, F. M., Russell, C. T., Smith, E. J., Southwood, D. J., and Tsurutani, B. T.: Cassini magnetometer observations during Saturn orbit insertion, Science, 307, 1266-1270, 2005.

Eriksson, A. I. and Wahlund, J. E.: Charging of the Freja satellite in the auroral zone, Ieee T. Plasma Sci., 34, 2038-2045, 2006.

Escoubet, C. P., Pedersen, A., Schmidt, R., and Lindqvist, P. A.: Density in the magnetosphere inferred from ISEE 1 spacecraft potential, J. Geophys. Res., 102, 17595-17609, 1997.

Espinosa, S. A., Southwood, D. J., and Dougherty, M. K.:
How can Saturn impose its rotation period in a noncorotating magnetosphere?, J. Geophys. Res., 108(A2), 1086, doi:10.1029/2001JA005084, 2003.

Giampieri, G., Dougherty, M. K., Smith, E. J., and Russell, C. T.: A regular period for Saturn's magnetic field that may track its internal rotation, Nature, 441, 62-64, doi:10.1038/nature04750, 2006.

Gurnett, D. A., Kurth, W. S., Kirchner, D. L., Hospodarsky, G. B., Averkamp, T. F., Zarka, P., Lecacheux, A., Manning, R., Roux, A., Canu, P., Cornilleau-Wehrlin, N., Galopeau, P., Meyer, A., Bostrom, R., Gustafsson, G., Wahlund, J. E., Ahlen, L., Rucker, H. O., Ladreiter, H. P., Macher, W., Woolliscroft, L. J. C., Alleyne, H., Kaiser, M. L., Desch, M. D., Farrell, W. M., Harvey, C. C., Louarn, P., Kellogg, P. J., Goetz, K., and Pedersen, A.: The Cassini radio and plasma wave investigation, Space Sci. Rev., 114, 395-463, 2004.

Gurnett, D. A., Persoon, A. M., Kurth, W. S., Groene, J. B., Averkamp, T. F., Dougherty, M. K., and Southwood, D. J.: The variable rotation period of the inner region of Saturn's plasma disk, Science, 316, 442-445, 2007.

Hill, T., Dessler, A., and Michel, F.: Configuration of the Jovian Magnetosphere, Geophys. Res. Lett., 1(1), 3-6, 1974.

Hill, T. W. and Michel, F. C.: Heavy-Ions from Galilean Satellites and Centrifugal-Distortion of Jovian Magnetosphere, J. Geophys. Res., 81, 4561-4565, 1976.

Khurana, K. K. and Schwarzl, H. K.: Global structure of Jupiter's magnetospheric current sheet, J. Geophys. Res., 110, A07227, doi:10.1029/2004JA010757, 2005.

Khurana, K. K., Mitchell, D. G., Arridge, C. S., Dougherty, M. K., Russell, C. T., Paranicas, C., Krupp, N., and Coates, A. J.: Sources of rotational signals in Saturn's magnetosphere, J. Geophys. Res., 114, A02211, doi:10.1029/2008JA013312, 2009.

Krimigis, S. M., Mitchell, D. G., Hamilton, D. C., Krupp, N., Livi, S., Roelof, E. C., Dandouras, J., Armstrong, T. P., Mauk, B. H., Paranicas, C., Brandt, P. C., Bolton, S., Cheng, A. F., Choo, T., Gloeckler, G., Hayes, J., Hsieh, K. C., Ip, W. H., Jaskulek, S., Keath, E. P., Kirsch, E., Kusterer, M., Lagg, A., Lanzerotti, L. J., LaVallee, D., Manweiler, J., McEntire, R. W., Rasmuss, W., Saur, J., Turner, F. S., Williams, D. J., and Woch, J.: Dynamics of Saturn's magnetosphere from MIMI during Cassini's orbital insertion, Science, 307, 1270-1273, 2005.

Krimigis, S. M., Sergis, N., Mitchell, D. G., Hamilton, D. C., and Krupp, N.: A dynamic, rotating ring current around Saturn, Nature, 450, 1050-1053, 2007.

Krupp, N., Lagg, A., Woch, J., Krimigis, S. M., Livi, S., Mitchell, D. G., Roelof, E. C., Paranicas, C., Mauk, B. H., Hamilton, D. C., Armstrong, T. P., and Dougherty, M. K.: The Saturnian plasma sheet as revealed by energetic particle measurements, Geophys. Res. Lett., 32, L20S03, doi:10.1029/2005GL022829, 2005.

Kurth, W. S., Lecacheux, A., Averkamp, T. F., Groene, J. B., and Gurnett, D. A.: A Saturnian longitude system based on a variable kilometric radiation period, Geophys. Res. Lett., 34, L02201, doi:10.1029/2006GL028336, 2007.

Lewis, G. R., André, N., Arridge, C. S., Coates, A. J., Gilbert, L. K., Linder, D. R., and Rymer, A. M.: Derivation of density and temperature from the cassini-huygens CAPS electron spectrometer, Planet. Space Sci., 56, 7, doi:10.1016/j.pss.2007.12.017, 2008.

Moncuquet, M., Lecacheux, A., Meyer-Vernet, N., Cecconi, B., and Kurth, W. S.: Quasi thermal noise spectroscopy in the 
inner magnetosphere of Saturn with Cassini/RPWS: Electron temperatures and density, Geophys. Res. Lett., 32, L20S02, doi:10.1029/2005GL022508, 2005.

Paranicas, C., Mitchell, D. G., Roelof, E. C., Brandt, P. C., Williams, D. J., Krimigis, S. M., and Mauk, B. H.: Periodic intensity variations in global ENA images of Saturn, Geophys Res Lett, 32, L21101, doi:10.1029/2005GL023656, 2005.

Pedersen, A.: Solar wind and magnetosphere plasma diagnostics by spacecraft electrostatic potential measurements, Ann. Geophys., 13, 118-129, 1995, http://www.ann-geophys.net/13/118/1995/.

Persoon, A. M., Gurnett, D. A., Kurth, W. S., Hospodarsky, G. B., Groene, J. B., Canu, P., and Dougherty, M. K.: Equatorial electron density measurements in Saturn's inner magnetosphere, Geophys. Res. Lett., 32, L23105, doi:10.1029/2005GL024294, 2005.

Persoon A. M., Gurnett, D. A., Kurth, W. S., and Groene, J. B.: A simple scale height model of the electron density in Saturn's plasma disk, Geophys. Res. Lett., 33, L18106, doi:10.1029/2006GL027090, 2006.

Pedersen, A., Lybekk, B., André, M., and Eriksson, A. I., Masson, A., Mozer, F. S., Lindqvist, P.-A., Décréau, P. M. E., Dandouras, I., Sauvaud, J.-A., Fazakerley, A., Taylor, A., Paschmann, G., Svenes, K. R., Torkar, K., and Whipple, E.: Electron density estimations derived from spacecraft potential measurements on Cluster in tenuous plasma regions, J. Geophys. Res., 113, A07S33, doi:10.1029/2007JA012636, 2008.

Richardson, J. D.: An Extended Plasma Model for Saturn, Geophys. Res. Lett., 22, 1177-1180, 1995.

Richardson, J. D., Eviatar, A., and Siscoe, G. L.: Satellite Tori at Saturn, J. Geophys. Res., 91, 8749-8755, 1986.

Richardson, J. D. and Sittler Jr., E. C.: A plasma density model for Saturn based on Voyager observations, J. Geophys. Res., 95, 12019-12031, 1990.

Saur, J., Mauk, B. H., Kaßner, A., and Neubauer, F. M.: A model for the azimuthal plasma velocity in Saturn's magnetosphere, J. Geophys. Res., 109, A05217, doi:10.1029/2003JA010207, 2004.

Sergis, N., Krimigis, S. M., Mitchell, D. G., Hamilton, D. C., Krupp, N., Mauk, B. M., Roelof, E. C., and Dougherty, M.: Ring current at Saturn: Energetic particle pressure in Saturn's equatorial magnetosphere measured with Cassini/MIMI, Geophys. Res. Lett., 34, L09102, doi:10.1029/2006GL029223, 2007.
Sergis, N., Krimigis, S. M., Mitchell, D. G., Hamilton, D. C., Krupp, N., Mauk, B. H., Roelof, E. C., and Dougherty, M.: Energetic particle pressure in Saturn's magnetosphere measured with the Magnetospheric Imaging Instrument on Cassini, J. Geophys. Res., 114, A02214, doi:10.1029/2008JA013774, 2009.

Sittler Jr., E. C., André, N., Blanc, M., Burger, M., Johnson, R. E., Coates, A., Rymer, A., Reisenfeld, D., Thomsen, M. F., Persoon, A., Dougherty, M., Smith, H. T., Baragiola, R. A., Hartle, R. E., Chornay, D., Shappirio, M. D., Simpson, D., McComas, D. J., and Young, D. T.: Ion and neutral sources and sinks within Saturns inner magnetosphere: Cassini results, Planet. Space Sci., 56, 3-18, 2008.

Southwood, D. J. and Kivelson, M. G.: Saturnian magnetospheric dynamics: Elucidation of a camshaft model, J. Geophys. Res. 112, A12222, doi:10.1029/2007JA012254, 2007.

Wahlund, J. E., Bostrom, R., Gustafsson, G., Gurnett, D. A., Kurth, W. S., Averkamp, T., Hospodarsky, G. B., Persoon, A. M., Canu, P., Pedersen, A., Desch, M. D., Eriksson, A. I., Gill, R., Morooka, M. W., and Andre, M.: The inner magnetosphere of Saturn: Cassini RPWS cold plasma results from the first encounter, Geophys. Res. Lett., 32, L20S09, doi:10.1029/2005GL022699, 2005.

Young, D. T., Berthelier, J. J., Blanc, M., Burch, J. L., Coates, A. J., Goldstein, R., Grande, M., Hill, T. W., Johnson, R. E., Kelha, V., McComas, D. J., Sittler, E. C., Svenes, K. R., Szego, K., Tanskanen, P., Ahola, K., Anderson, D., Bakshi, S., Baragiola, R. A., Barraclough, L., Black, R. K., Bolton, S., Booker, T., Bowman, R., Casey, P., Crary, F. J., Delapp, D., Dirks, G., Eaker, N., Funsten, H., Furman, J. D., Gosling, J. T., Hannula, H., Holmlund, C., Huomo, H., Illiano, J. M., Jensen, P., Johnson, M. A., Linder, D. R., Luntama, T., Maurice, S., McCabe, K. P., Mursula, K., Narheim, B. T., Nordholt, J. E., Preece, A., Rudzki, J., Ruitberg, A., Smith, K., Szalai, S., Thomsen, M. F., Viherkanto, K., Vilppola, J., Vollmer, T., Wahl, T. E., Wuest, M., Ylikorpi, T., and Zinsmeyer, C.: Cassini Plasma Spectrometer investigation, Space Sci. Rev., 114, 1-112, 2004. 